\title{
Evidence of dynamical dipole excitation in the fusion-evaporation of the ${ }^{40} \mathrm{Ca}+{ }^{152} \mathrm{Sm}$ heavy system
}

\author{
C. Parascandolo, ${ }^{1}$ D. Pierroutsakou, ${ }^{1, *}$ R. Alba,${ }^{2}$ A. Del Zoppo,${ }^{2}$ C. Maiolino, ${ }^{2}$ D. Santonocito, ${ }^{2}$ C. Agodi, ${ }^{2}$ V. Baran, $, 2,3,4$ \\ A. Boiano, ${ }^{1}$ M. Colonna, ${ }^{2}$ R. Coniglione, ${ }^{2}$ E. De Filippo, ${ }^{5}$ M. Di Toro, ${ }^{2,6}$ U. Emanuele, ${ }^{7}$ F. Farinon, ${ }^{8}$ A. Guglielmetti, ${ }^{9,10}$ \\ G. Inglima, ${ }^{1,11}$ M. La Commara, ${ }^{1,11}$ B. Martin, ${ }^{1,11}$ C. Mazzocchi, ${ }^{9,10}$ M. Mazzocco, ${ }^{12,13}$ C. Rizzo, ${ }^{2,6}$ M. Romoli, ${ }^{1}$ \\ M. Sandoli, ${ }^{1,11}$ C. Signorini, ${ }^{12,13}$ R. Silvestri, ${ }^{1,11}$ F. Soramel, ${ }^{12,13}$ E. Strano, ${ }^{12,13}$ D. Torresi, ${ }^{12,13}$ A. Trifirò, ${ }^{7}$ and M. Trimarchi ${ }^{7}$ \\ ${ }^{1}$ INFN, Sezione di Napoli, via Cintia, 80126 Napoli, Italy \\ ${ }^{2}$ INFN, Laboratori Nazionali del Sud, via Santa Sofia 62, 95123 Catania, Italy \\ ${ }^{3}$ Physics Faculty, University of Bucharest, Romania \\ ${ }^{4}$ NIPNE-HH, 077125 Magurele, Romania \\ ${ }^{5}$ INFN, Sezione di Catania, via Santa Sofia 64, 95123 Catania, Italy \\ ${ }^{6}$ Dipartimento di Fisica e Astronomia, Università di Catania, via Santa Sofia 64, 95123 Catania, Italy \\ ${ }^{7}$ Dipartimento di Fisica e di Scienze della Terra, Università degli Studi di Messina and INFN Gruppo Collegato di Messina, \\ viale F. Stagno D'Alcontres 31, 98166 Sant'Agata, Messina, Italy \\ ${ }^{8}$ GSI, Planckstrasse 1, 64291 Darmstadt, Germany \\ ${ }^{9}$ Dipartimento di Fisica, Università di Milano, via Celoria 16, 20133 Milano, Italy \\ ${ }^{10}$ INFN, Sezione di Milano, via Celoria 16, 20133 Milano, Italy \\ ${ }^{11}$ Dipartimento di Fisica, Università di Napoli "Federico II", via Cintia, 80126 Napoli, Italy \\ ${ }^{12}$ Dipartimento di Fisica e Astronomia, Università di Padova, via Francesco Marzolo 8, 35131 Padova, Italy \\ ${ }^{13}$ INFN, Sezione di Padova, via Francesco Marzolo 8, 35131 Padova, Italy
}

(Received 19 January 2016; published 29 April 2016)

\begin{abstract}
The excitation of the dynamical dipole mode along the fusion path was investigated for the first time in the formation of a heavy compound nucleus in the $A \sim 190$ mass region. The compound nucleus was formed at identical conditions of excitation energy and spin from two entrance channels: the charge-asymmetric ${ }^{40} \mathrm{Ca}+{ }^{152} \mathrm{Sm}$ and the nearly charge-symmetric ${ }^{48} \mathrm{Ca}+{ }^{144} \mathrm{Sm}$ at $E_{\text {lab }}=11$ and $10.1 \mathrm{MeV} /$ nucleon, respectively. High-energy $\gamma$ rays and light charged particles were measured in coincidence with evaporation residues by means of the MEDEA multidetector array (Laboratori Nazionali del Sud, Italy) coupled to four parallel plate avalanche counters. The charged particle multiplicity spectra and angular distributions were used to pin down the average excitation energy, the average mass, and the average charge of the compound nucleus. The $\gamma$-ray multiplicity spectrum and angular distribution related to the nearly charge-symmetric channel were employed to obtain new data on the giant dipole resonance in the compound nucleus. The dynamical dipole mode excitation in the charge-asymmetric channel was evidenced, in a model-independent way, by comparing the $\gamma$-ray multiplicity spectra and angular distributions of the two entrance channels with each other. Calculations of the dynamical dipole mode in the ${ }^{40} \mathrm{Ca}+{ }^{152} \mathrm{Sm}$ channel, based on a collective bremsstrahlung analysis of the reaction dynamics, are presented. Possible interesting implications in the superheavy-element quest are discussed.
\end{abstract}

\section{DOI: 10.1103/PhysRevC.93.044619}

\section{INTRODUCTION}

During the early stages of a charge-asymmetric heavy-ion collision the charge equilibration can be reached through incoherent exchange of nucleons between the colliding ions or through the excitation of a collective dipole oscillation along the symmetry axis of the highly deformed composite system, the dinucleus. This is the so-called dynamical dipole (DD) mode or preequilibrium giant dipole resonance, foreseen in various theoretical works [1-9]. The charge equilibration mechanism between the reaction partners is influenced by different parameters like charge and mass asymmetry, impact parameter, collision energy, and mass of the colliding ions. If the collective dipole mode develops in the entrance channel, preequilibrium $\gamma$ radiation can be emitted during the dinuclear phase of the reaction in addition to that coming from the statistical giant dipole resonance (GDR) excited in the

*Corresponding author: pierroutsakou@na.infn.it compound nucleus. This preequilibrium radiation carries out relevant information about the first stages of the collision and constitutes a powerful probe to get insight into: (a) the reaction dynamics and, particularly, on the charge equilibration mechanism; (b) the density dependence of the symmetry energy in the nuclear matter equation of state (EOS) at densities lower than the saturation one, where the DD is active. Furthermore, it has been proposed as a possible fast cooling mechanism of the composite system on the way to fusion, favoring the compound nucleus survival against statistical fission and, thus, the formation of a superheavy element [8,10].

Experimentally, the DD excitation and subsequent $\gamma$ decay has been observed in deep inelastic $[6,11,12]$ and fusion-evaporation heavy-ion collisions [12-20]. In some of the above-cited works dealing with fusion-evaporation experiments this has been reached by employing a modelindependent method, the so-called difference technique, that consists in (a) forming the same compound nucleus at identical conditions of excitation energy and spin from two entrance 
channels, a nearly charge-symmetric and a charge-asymmetric one, and (b) obtaining the difference between the $\gamma$-ray spectra of the two channels for fusion-evaporation events. The resulting difference showed an excess of yield in the more charge-asymmetric channel, ascribed to a preequilibrium effect, namely the DD excitation. Following the Bohr hypothesis, if the $\gamma$ spectra were related to the sole compound nucleus decay, they would have been independent of the entrance channel and no extra yield would have been observed.

Besides the fact that the observation of a $\gamma$-ray excess in the channel with the higher charge asymmetry denotes by itself a preequilibrium phenomenon, further proofs of its prompt nature were given in our previous works [15-18]. In those experiments the DD excitation was investigated in a ${ }^{132} \mathrm{Ce}$ composite system, formed through the ${ }^{32,36} \mathrm{~S}+{ }^{100,96}$ Mo and ${ }^{36,40} \mathrm{Ar}+{ }^{96,92} \mathrm{Zr}$ fusion-evaporation reactions at incident energies $E_{\mathrm{lab}}=6,9$, and $16 \mathrm{MeV} /$ nucleon. The $\mathrm{DD} \gamma$ radiation was isolated by means of the difference technique previously described. By studying the energy spectrum of the DD $\gamma$-ray multiplicity and its angular distribution, the following results were obtained from this campaign of measurements. (i) The DD centroid energy was found to be lower than that of the ground-state GDR, indicating a high deformation of the emitting source $[15,17,18]$. This was in agreement with theoretical predictions for an oscillation along the symmetry axis of a deformed dinuclear shape during the early moments of the reaction, when complete equilibrium is still not achieved [3,4,6,8-10,21]. (ii) The observed anisotropy of the DD $\gamma$-ray angular distribution with respect to the beam direction was found to be larger than that corresponding to emission of statistical GDR $\gamma$ rays from a rotating hot compound nucleus. This was interpreted as the signature of a preferential oscillation axis of the DD triggered at the early stage of the fusion path along an axis that has not rotated much on the reaction plane during the DD lifetime $[17,18]$. Also this observation was in agreement with calculations done for low-impact parameters leading to fusion-evaporation events [21]. (iii) The DD excitation function showed a "rise and fall" behavior with a maximum at $E_{\text {lab }}=9 \mathrm{MeV} /$ nucleon. Although calculations obtained in the framework of a BoltzmannNordheim-Vlasov (BNV) transport model suggested a similar behavior [8], the "rise and fall" trend with incident energy was predicted to be smoother than that found in the data [18].

A second campaign of experiments was performed to probe the DD excitation in the same composite system, ${ }^{132} \mathrm{Ce}$, formed through an entrance channel with a larger mass asymmetry and a lower charge asymmetry than previously: the ${ }^{16} \mathrm{O}+{ }^{116} \mathrm{Sn}$ reaction at $E_{\mathrm{lab}}=8.1,12$, and $15.6 \mathrm{MeV} /$ nucleon $[19,20]$. In this campaign a different method was used: The DD radiation was extracted by subtracting the $\gamma$-ray multiplicity spectrum of the compound nucleus, calculated by means of the statistical model code CASCADE [22], from the ${ }^{16} \mathrm{O}+{ }^{116} \mathrm{Sn}$ experimental $\gamma$ spectrum. The CASCADE calculation used in the comparison was tested with a near-symmetric reaction, leading to the same compound nucleus ${ }^{132} \mathrm{Ce}$.

By comparing the results of the two campaigns with each other and with the theoretical predictions, a rather complex scenario emerged that called for further investigation to clarify different aspects related to the DD excitation. On the one hand, calculations, while being able to describe the phenomenon and absolute values of some observables, are not able to fully reproduce the existing experimental findings $[18,19]$. On the other hand, few experimental results exist reporting on the DD $\gamma$ multiplicity and on its angular distribution, which can be directly compared with calculations. These elements called for more experimental efforts to disentangle the influence of each reaction parameter on the DD features and to provide severe constraints to the theoretical models.

In the present work we give evidence for the first time of DD excitation in a heavy dinucleus in the $A \sim 190$ mass region, by isolating its prompt $\gamma$ radiation in fusion-evaporation events. To that end, as in our previous works [15-18], we use here the difference technique by employing two reactions: the charge-asymmetric ${ }^{40} \mathrm{Ca}+{ }^{152} \mathrm{Sm}$ and the nearly chargesymmetric ${ }^{48} \mathrm{Ca}+{ }^{144} \mathrm{Sm}$ at $E_{\mathrm{lab}}=11$ and $10.1 \mathrm{MeV} /$ nucleon, respectively. The multiplicity spectra and angular distribution of the light charged particles detected in coincidence with the evaporation residues are used to determine the average mass, average charge, and average excitation energy of the compound nucleus and to ensure us that the same compound nucleus at identical conditions is formed in the two reactions. First, we examine the $\gamma$-ray multiplicity spectrum and $\gamma$-ray angular distribution of the nearly charge-symmetric channel for evaporation events to obtain information on the statistical GDR in the highly excited compound nucleus. Then, by comparing the $\gamma$-ray multiplicity spectra and angular distributions of the two reactions with each other, we evidence the preequilibrium component originated from the DD excitation in the dinucleus of the charge-asymmetric system. The extracted DD $\gamma$-ray multiplicity spectrum and angular distribution are compared with the theoretical ones, obtained in the framework of a BNV transport model based on a collective bremsstrahlung analysis of the reaction dynamics [8,21]. The observation of DD $\gamma$ radiation in the evaporation channel of such a heavy composite system, heavier than those studied so far, could have interesting implications in the superheavy-element quest.

The paper is organized as follows. In Sec. II the experimental techniques are presented. The analysis results are discussed in Sec. III: in part A the light charged particles data are used to define the compound nucleus mass, charge, and excitation energy; in part B the multiplicity spectra and angular distributions of the $\gamma$ rays are examined. In Sec. III B 1 the spectrum of the nearly charge-symmetric channel is analyzed in the framework of the statistical model while in Sec. III B 2 the preequilibrium $\gamma$-ray component is extracted in the chargeasymmetric channel. The $\gamma$-ray angular distributions of both entrance channels and of the DD are shown and discussed in Sec. III B 3. The BNV calculations are compared with the experimental findings in Sec. IV, where also a discussion of the obtained results is done. Finally, in Sec. V, conclusions and perspectives are given.

\section{EXPERIMENT}

In Table I, we summarize the entrance channel and compound nucleus relevant quantities for the studied reactions. The entrance channel dipole moment $D(t=0)$ is given by the 
TABLE I. Reaction, incident energy, entrance channel dipole moment $D(t=0)$, and mass asymmetry $\Delta$. Compound nucleus $\mathrm{CN}_{\mathrm{CF}}$ and its excitation energy $E_{\mathrm{CF}}^{*}$ for complete fusion. $\mathrm{CN}, E^{*}$ refer to the compound nucleus after preequilibrium particle emission as derived from the charged-particle spectra analysis in Sec. III.A. The excitation energies were calculated at midtarget position. $J_{B_{f}=0}$ is the critical angular momentum at which the fission barrier vanishes for the complete fusion compound nucleus and in parentheses for the compound nucleus after preequilibrium particle emission [24].

\begin{tabular}{lcccccrr}
\hline \hline Reaction & $\begin{array}{c}E_{\text {lab }} \\
(\mathrm{MeV} / \text { nucleon })\end{array}$ & $\begin{array}{c}D(t=0) \\
(\mathrm{fm})\end{array}$ & $\Delta$ & $\mathrm{CN}_{\mathrm{CF}}$ & $\begin{array}{c}E_{\mathrm{CF}}^{*} \\
(\mathrm{MeV})\end{array}$ & $\begin{array}{c}\mathrm{CN} \\
J_{B_{f}=0} \\
(\hbar)\end{array}$ & $\begin{array}{c}E^{*} \\
(\mathrm{MeV})\end{array}$ \\
\hline${ }^{40} \mathrm{Ca}+{ }^{152} \mathrm{Sm}$ & 11.0 & 30.6 & 0.22 & ${ }^{192} \mathrm{~Pb}$ & 258.75 & $189 \mathrm{Hg}$ & $219.6 \pm 6.8$ \\
$\mathrm{Ca}+{ }^{144} \mathrm{Sm}$ & 10.1 & 5.3 & 0.18 & ${ }^{192} \mathrm{~Pb}$ & 257.49 & $189 \mathrm{Hg}$ & $220.5 \pm 6.6$ \\
\hline \hline
\end{tabular}

relation

$$
\begin{aligned}
D(t=0) & =\frac{N Z}{A}\left|R_{Z}(t=0)-R_{N}(t=0)\right| \\
& =\frac{r_{0}\left(A_{p}^{1 / 3}+A_{t}^{1 / 3}\right)}{A} Z_{p} Z_{t}\left|\frac{N_{p}}{Z_{p}}-\frac{N_{t}}{Z_{t}}\right|,
\end{aligned}
$$

$R_{Z}$ and $R_{N}$ being the center-of-mass coordinates of protons and neutrons, respectively. $A=A_{p}+A_{t}$ is the mass of the composite system, $N=N_{p}+N_{t}\left(Z=Z_{p}+Z_{t}\right)$ its neutron (proton) number while the subscripts $p$ and $t$ refer to the projectile and target, respectively. For the $D(t=0)$ calculation, $r_{0}=1.2 \mathrm{fm}$ was used to deduce the nuclear radii. The entrance channel mass asymmetry $\Delta=\frac{\left|A_{t}^{1 / 3}-A_{p}^{1 / 3}\right|}{A_{t}^{1 / 3}+A_{p}^{1 / 3}}$ of the two reactions was very similar. The ${ }^{189} \mathrm{Hg}$ compound nucleus was formed after preequilibrium particle emission at identical excitation energy in both reactions, as demonstrated in Sec. III A, and with the same spin distribution according to PACE2 [23] calculations. The critical angular momentum for the compound nucleus formation, $J_{B_{f}=0}$, is shown in Table I and corresponds to the angular momentum where the fission barrier vanishes [24].

The ${ }^{40} \mathrm{Ca}\left({ }^{48} \mathrm{Ca}\right)$ pulsed beam, provided by the Superconducting Cyclotron of the Laboratori Nazionali del Sud (Italy), impinged on a $1 \mathrm{mg} / \mathrm{cm}^{2}$ thick self-supporting ${ }^{152} \mathrm{Sm}\left({ }^{144} \mathrm{Sm}\right)$ target enriched to $98.4 \%$ (93.8\%). The ${ }^{40} \mathrm{Ca}\left({ }^{48} \mathrm{Ca}\right)$ beam consisted of bunches with a 120-ns separation and with a bunch standard deviation of $\sigma=1.6$ (1.2) ns. Beam current was about $1 \mathrm{nA}$ for both reactions.

The $\gamma$ rays and the light charged particles were detected by using the 180 barium fluoride $\left(\mathrm{BaF}_{2}\right)$ modules of the MEDEA experimental apparatus installed at Laboratori Nazionali del Sud (Italy), that covers the polar angular range between $\theta_{\text {lab }}=$ $30^{\circ}$ and $\theta_{\mathrm{lab}}=170^{\circ}$ and the full range in the azimuthal angle $\phi$ (for more details see Ref. [25]). The crystals are arranged in eight rings, each of them located at a fixed angle. The MEDEA ball has an inner radius of $22 \mathrm{~cm}$ and covers a total solid angle of $3.7 \pi \mathrm{sr}$. The apparatus operates under vacuum inside a large scattering chamber to allow a simultaneous detection of $\gamma$ rays and light charged particles. Only seven among the eight rings were used in the present experiment, positioned at $\theta_{\text {lab }}=$ $51.5^{\circ}, 68.1^{\circ}, 82.8^{\circ}, 97.1^{\circ}, 111.9^{\circ}, 128.5^{\circ}$, and $159.7^{\circ}$.

The photomultiplier output of each $\mathrm{BaF}_{2}$ module was split into four differently weighted signals. One was used for timing, two for energy integration in two different dynamical ranges (about 30 and about $200 \mathrm{MeV}$ full scale, respectively), and one to integrate the $\mathrm{BaF}_{2}$ fast light emission component.
The combination of this last information with the energy one allows us to obtain a shape analysis of the $\mathrm{BaF}_{2}$ signal. The discrimination between $\gamma$ rays, light charged particles, and neutrons was performed by combining this pulse shape analysis with a time-of-flight (TOF) measurement, between each $\mathrm{BaF}_{2}$ detector and the radiofrequency signal of the cyclotron.

An example of the discrimination of $\gamma$ rays from neutrons and charged particles is shown in Fig. 1. Panel (a) shows the bidimensional plot of fast component vs total energy for $\mathrm{a} \mathrm{BaF}_{2}$ at $\theta_{\mathrm{lab}}=51.5^{\circ}$ in the low-gain energy range for the reaction ${ }^{40} \mathrm{Ca}+{ }^{152} \mathrm{Sm}$. For the same detector, panel (b) displays the bidimensional plot time of flight vs total energy, while panel (c) illustrates the latter plot only for events included in the contour of panel (a). By applying therefore a contour in panel (a), an unambiguous discrimination between $\gamma$ rays, protons, and $\alpha$ particles is achieved. Low-energy neutrons $\left(E_{n} \leqslant 20 \mathrm{MeV}\right)$, giving the same pulse shape as $\gamma$ rays, are separated from $\gamma$ rays by the TOF measurement, as can be seen in panel (c) of Fig. 1 [26]; high-energy neutrons $\left(E_{n}>20 \mathrm{MeV}\right)$, giving a pulse shape similar to the proton one, are separated from the $\gamma$ rays in the fast component vs total energy plot [27]. Thus, the $\gamma$ rays retained in the analysis are obtained by applying both the contours shown in panels (a) and (c). The same procedure was applied for the high-gain energy range for $\gamma$ rays and for the light charged particles by using the appropriate contours.

The energy calibration of the $\gamma$-ray detectors was obtained by using the composite sources of ${ }^{241} \mathrm{Am}+{ }^{9} \mathrm{Be}\left(E_{\gamma}=\right.$ $4.43 \mathrm{MeV})$ and ${ }^{238} \mathrm{Pu}+{ }^{13} \mathrm{C}\left(E_{\gamma}=6.13 \mathrm{MeV}\right)$ and the 15.1$\mathrm{MeV} \gamma$ rays from the $p+{ }^{12} \mathrm{C}$ reaction at $E_{p}=25 \mathrm{MeV}$. The charged particle calibration was deduced from the $\gamma$ calibration as described elsewhere [28]. The time stability of the energy calibration was checked during the experiment by monitoring after each run the stability of the peak corresponding to a radioactive source.

The fusion-evaporation residues were detected by four parallel-plate avalanche counters (PPACs) located symmetrically around the beam direction at $70 \mathrm{~cm}$ from the target in an annular configuration. The PPACs were centered at an angle $\theta_{\mathrm{lab}}=7^{\circ}$ with respect to the beam direction, subtending $7^{\circ}$ in $\theta$ and covering a total solid angle of 0.089 sr. PACE 2 calculations show that the evaporation residue angular distribution has a maximum at $\theta_{\mathrm{lab}}=4.5^{\circ}$ and extends up to $\theta_{\mathrm{lab}}=16^{\circ}$ for both reactions. That ensures us that we selected experimentally the same evaporation residues in both reactions (about $70 \%$ of the whole evaporation residue cross section), avoiding thus any difference that could influence our results on the DD. 

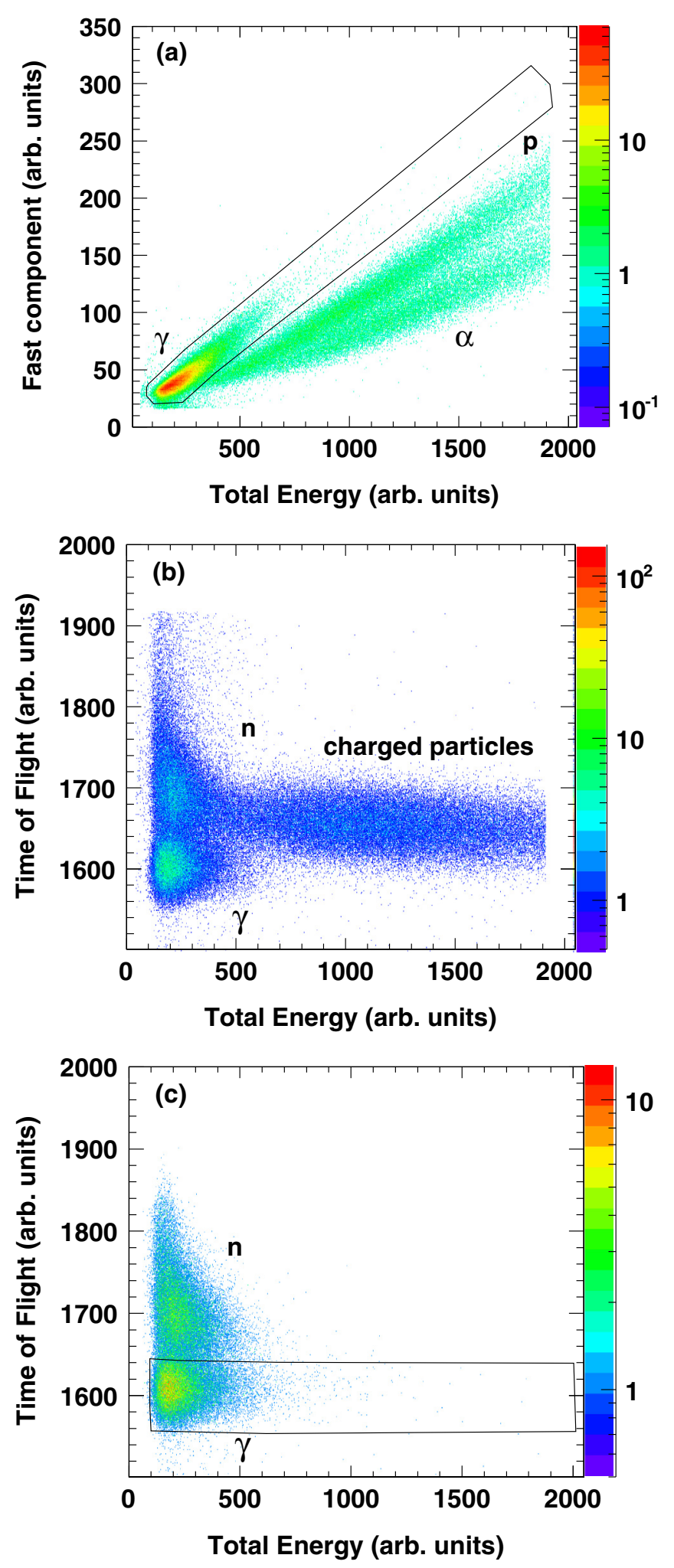

FIG. 1. (a) Fast component vs total energy and (b) time of flight

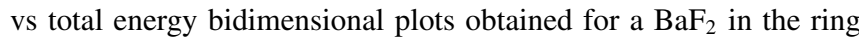
centered at $\theta_{\text {lab }}=51.5^{\circ}$ in the low-gain energy range for the reaction ${ }^{40} \mathrm{Ca}+{ }^{152} \mathrm{Sm}$; (c) time of flight vs total energy of the same detector is shown for events included in the contour of panel (a). The $\gamma$ rays retained in the analysis are obtained by applying both of the contours shown in panels (a) and (c).
The PPACs provided the TOF signal with respect to the radiofrequency signal of the cyclotron and the energy loss $(\Delta E)$ of the reaction products. Panel (a) of Fig. 2 presents the energy loss $\Delta E$ vs time of flight bidimensional plot, obtained with one of the PPACs for the ${ }^{40} \mathrm{Ca}+{ }^{152} \mathrm{Sm}$ reaction. A TOF calibration was achieved by considering the distance between the elastic peaks observed during runs characterized by fixed delays in the radiofrequency signal, when the electronic threshold of the $\Delta E$ signal was suitably lowered. The locus at small TOF and low $\Delta E$ (marked with the number 1 in the figure) indicates events where the projectile was elastically scattered by the target. These events are discarded by the electronic threshold of the PPACs to reduce the acquisition dead time. The locus characterized by higher $\Delta E$ and longer TOF (marked with the number 2) corresponds to peripheral events going from the elastic peak of the projectile (low $\Delta E$ ) to the elastic peak of the target nucleus (highest $\Delta E$ ) and/or fission events. The region with the longest TOF (marked with the number 3) corresponds to the evaporation residues. Panel (b) of Fig. 2 displays the velocity distribution of the evaporation residues that were retained in the analysis. The arrows correspond to the average velocity of the distribution, $V_{C N}$ and to the center-of-mass velocity $V_{\text {c.m. }}$, which is the complete fusion value of $V_{C N}$ for full linear momentum transfer.

Downscaled single PPAC events together with coincidence events between a PPAC and at least one fired $\mathrm{BaF}_{2}$ scintillator were collected during the experiment. A coincidence event was accepted if the deposited energy in a $\mathrm{BaF}_{2}$ detector was greater than $\sim 5.5 \mathrm{MeV}$ for $\gamma$ rays. The coincidence request eliminated any cosmic-ray contamination of the $\gamma$-ray spectra.

\section{ANALYSIS AND RESULTS}

The experiment was designed in such a way to form the same compound nucleus at identical excitation energy from the two entrance channels by taking into account incomplete fusion events [29]. These events are characterized by emission of preequilibrium light particles that reduces the compound nucleus average mass, average charge, and average excitation energy and cannot be discarded in the TOF spectrum of the reaction products because they have overlapping velocity distributions with those of the complete fusion events [30].

In the present work, the average excitation energy, the average mass, and the average charge of the composite system, after preequilibrium particle emission, were evaluated by studying the energy spectra of the light charged particles detected in coincidence with evaporation residues, while the preequilibrium neutron emission was estimated from our proton data and from existing neutron emission studies (see Ref. [31] and references therein).

\section{A. Light charged particle spectra}

The laboratory proton and $\alpha$-particle multiplicity spectra obtained with $\mathrm{BaF}_{2}$ scintillators centered at polar angles $51.5^{\circ}<\theta_{\text {lab }}<159.7^{\circ}$, in coincidence with the evaporation residues, are reported in Figs. 3 and 4, respectively.

These spectra were analyzed by means of a moving source fit in which the particles were assumed to be 

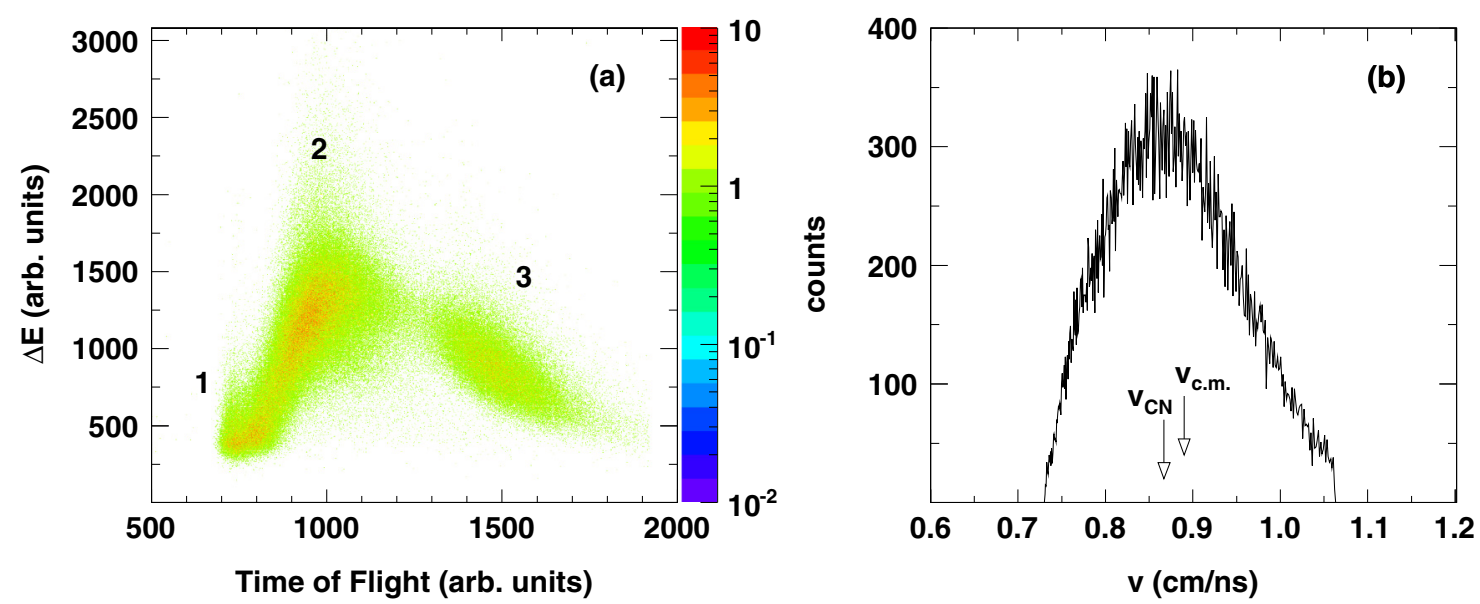

FIG. 2. (a) Energy loss $\Delta E$ vs time of flight bidimensional plot obtained with one of the PPACs for the ${ }^{40} \mathrm{Ca}+{ }^{152} \mathrm{Sm}$ reaction. The numbers indicate different physical processes (see text). The evaporation residues are marked with the number 3. (b) Velocity distribution of the evaporation residues retained in the analysis. The arrows correspond to the average velocity of the distribution, $V_{C N}$, and the center-of-mass velocity $V_{\text {c.m. }}$, which is the complete fusion value of $V_{C N}$ for full linear momentum transfer. In the figure, the velocity is not corrected for the energy loss of the evaporation residues in the target.
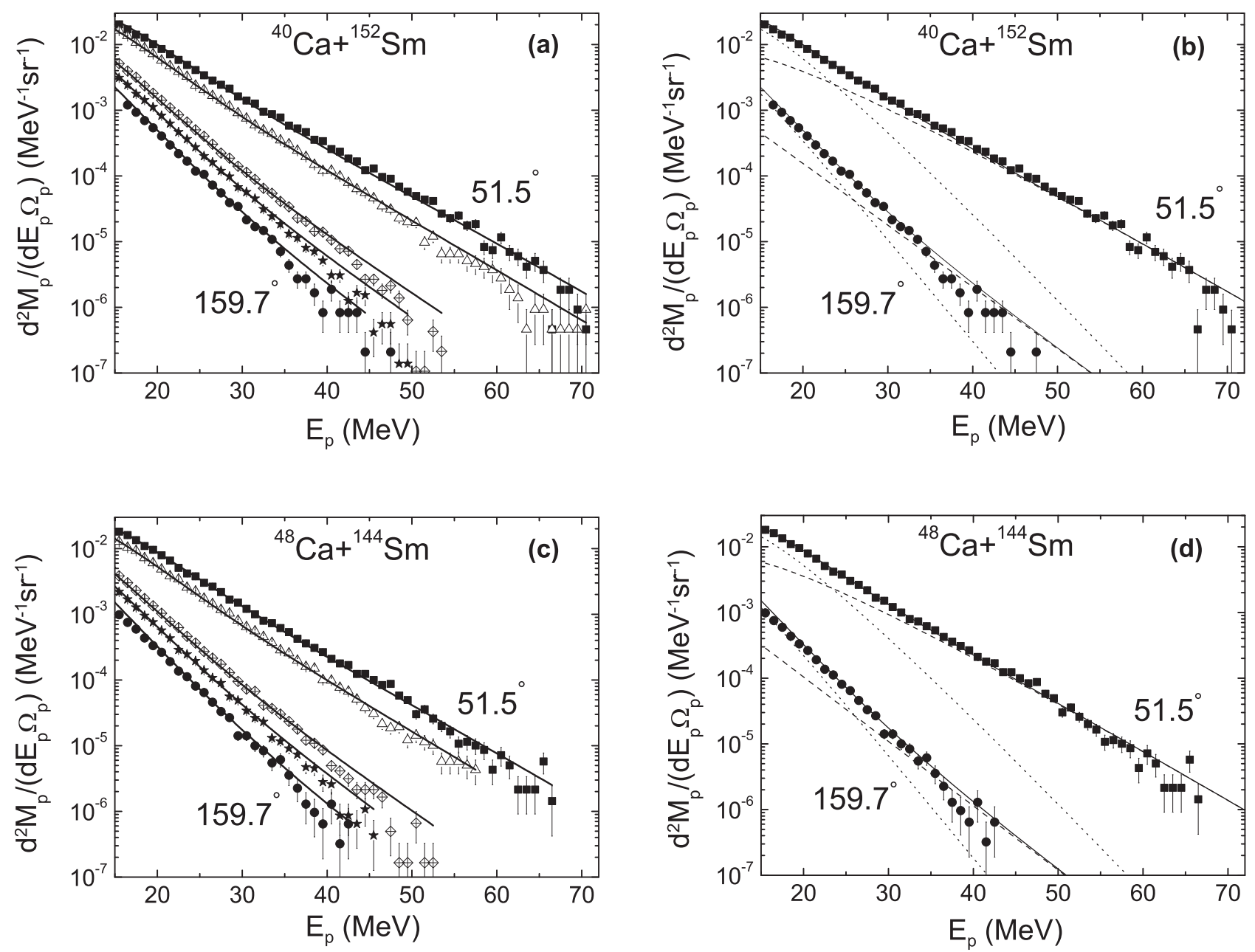

FIG. 3. (a), (c) Laboratory proton multiplicity spectra obtained at various angles in coincidence with the evaporation residues. For both reactions the angles are (from top to bottom) $51.5^{\circ}, 68.1^{\circ}, 111.9^{\circ}, 128.5^{\circ}$, and $159.7^{\circ}$. The solid lines show the result of the simultaneous fit with two sources described in the text. (b),(d) Laboratory proton multiplicity spectra at two angles. The solid lines are the result of the fit with two sources. The dashed and dotted lines represent the intermediate-velocity source and the slow source components, respectively. 

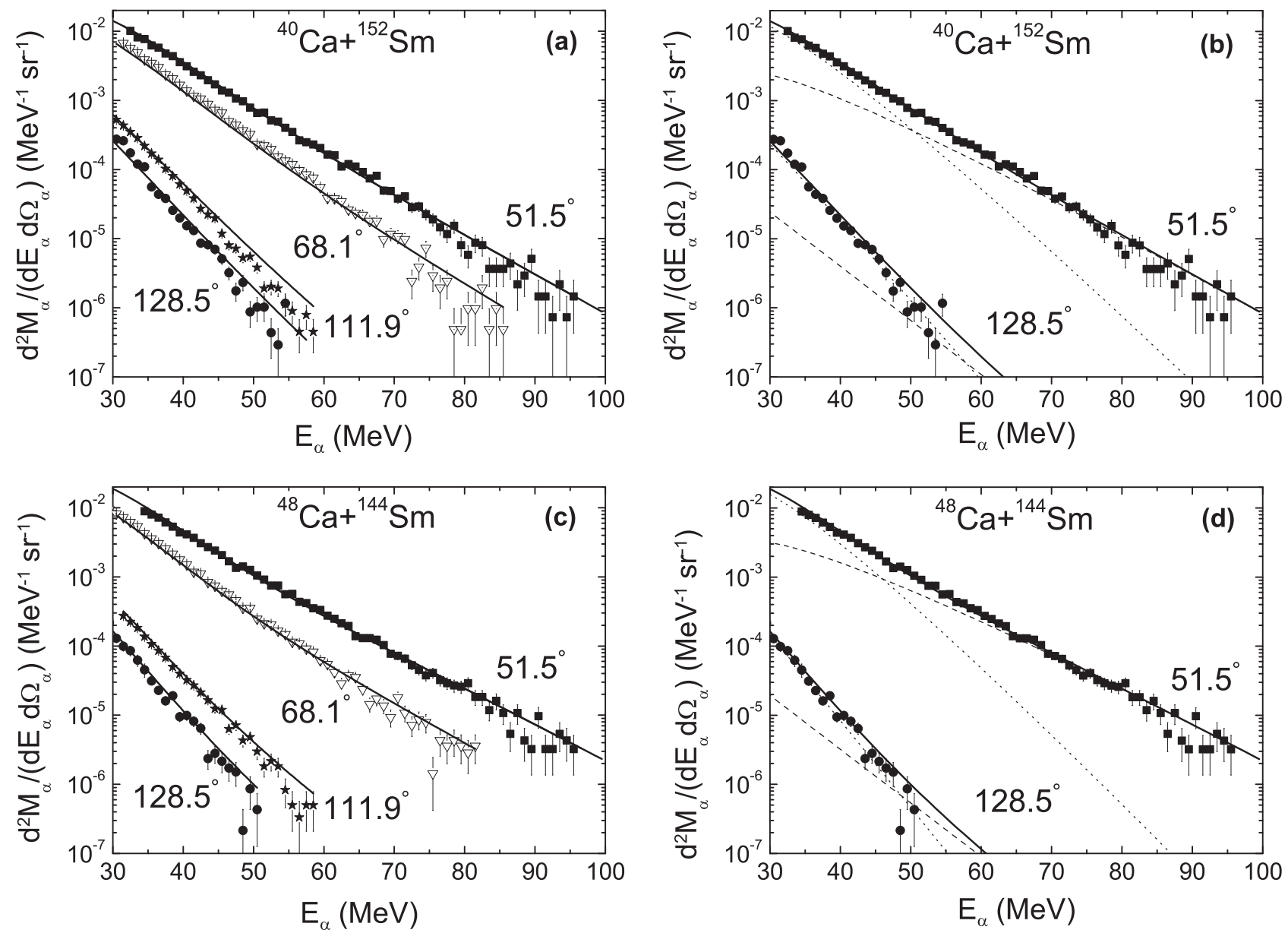

FIG. 4. As in Fig. 3 for $\alpha$ particles.

emitted isotropically from two moving sources: a slow source describing the statistical evaporation from the hot compound nucleus and an intermediate-velocity (between the compound nucleus and the projectile velocity) source related to the preequilibrium particles emitted by the composite system before thermalization.

The energy distribution of the evaporated particles was parametrized, in the source rest frame, adopting a surface-type Maxwellian distribution given by

$$
\left(\frac{d^{2} M}{d \Omega d E}\right)_{\mathrm{sl}}=\frac{M_{\mathrm{sl}}}{4 \pi T_{\mathrm{sl}}^{2}}\left(E-E_{c}\right) \exp \left[-\frac{\left(E-E_{c}\right)}{T_{\mathrm{sl}}}\right]
$$

while the distribution of the preequilibrium particles was taken to be that for volume emission from a thermal source:

$$
\left(\frac{d^{2} M}{d \Omega d E}\right)_{\mathrm{int}}=\frac{M_{\mathrm{int}}}{2\left(\pi T_{\mathrm{int}}\right)^{3 / 2}} \sqrt{E-E_{c}} \exp \left[-\frac{\left(E-E_{c}\right)}{T_{\mathrm{int}}}\right],
$$

where $E$ is the particle energy, $E_{c}$ is the Coulomb barrier for particle emission, $T_{i}$ ( $i$ is for sl or int) is the apparent source temperature, and $M_{i}$ is the multiplicity of the particles emitted from the $M_{i}$ source. Both Maxwellian distributions were transformed in the laboratory reference frame using the relation

$$
\left(\frac{d^{2} M}{d \Omega d E}\right)_{\mathrm{lab}}=\sqrt{\frac{E_{\mathrm{lab}}}{E^{\prime}}}\left(\frac{d^{2} M}{d \Omega d E}\right)_{E=E^{\prime}},
$$

where the particle energy $E^{\prime}$ in the source reference frame is given by

$$
E^{\prime}=E_{\mathrm{lab}}+E_{s}-2 \sqrt{E_{\mathrm{lab}} E_{s}} \cos \theta_{\mathrm{lab}},
$$

where $E_{s}$ is the energy of a particle moving with the source velocity, assumed to be parallel to the beam axis and $\theta_{\text {lab }}$ is the observation angle of the particle.

The evaporative source velocity was fixed to the value $v_{\mathrm{sl}}=$ $0.937 \mathrm{~cm} / \mathrm{ns}$ and $1.028 \mathrm{~cm} / \mathrm{ns}$ for the system ${ }^{40} \mathrm{Ca}+{ }^{152} \mathrm{Sm}$ and ${ }^{48} \mathrm{Ca}+{ }^{144} \mathrm{Sm}$, respectively and was derived from the average value of the evaporation residue velocity distributions $V_{C N}$ [see panel (b) of Fig. 2] correcting for the energy loss in half thickness of the target. We observed that the same Coulomb barrier values could be employed to fit the particle spectra. We used $E_{c}=6$ and $14 \mathrm{MeV}$ for protons and $\alpha$ particles, respectively. We remark that the sensitivity of the fit to reasonable changes of the Coulomb barrier values is small. The remaining five parameters were considered as free variables in the fitting procedure.

The result of the simultaneous fit is shown with solid lines in panels (a) and (c) of Figs. 3 and 4 for protons and $\alpha$ particles, 
TABLE II. Multiplicities, temperatures, and velocities of the slow and of the intermediate-velocity sources extracted from the moving source fit for protons emitted during the ${ }^{40} \mathrm{Ca}+{ }^{152} \mathrm{Sm}$ and ${ }^{48} \mathrm{Ca}+{ }^{144} \mathrm{Sm}$ fusion-evaporation reactions.

\begin{tabular}{lcccccrc}
\hline \hline Reaction & $E_{c}(\mathrm{MeV})$ & $v_{\mathrm{sl}}(\mathrm{cm} / \mathrm{ns})$ & $M_{\mathrm{sl}}$ & $T_{\mathrm{sl}}(\mathrm{MeV})$ & $v_{\text {int }}(\mathrm{cm} / \mathrm{ns})$ & $M_{\text {int }}$ \\
\hline${ }^{40} \mathrm{Ca}+{ }^{152} \mathrm{Sm}$ & 6 & 0.937 & $2.254 \pm 0.052$ & $2.860 \pm 0.026$ & $1.730 \pm 0.058$ & $0.786 \pm 0.060$ & $5.141 \pm 0.089$ \\
${ }^{48} \mathrm{Ca}+{ }^{144} \mathrm{Sm}$ & 6 & 1.028 & $1.772 \pm 0.057$ & $2.866 \pm 0.028$ & $1.843 \pm 0.071$ & $0.693 \pm 0.062$ & $5.008 \pm 0.104$ \\
\hline \hline
\end{tabular}

respectively. The relative contributions of the two sources are reported in panels (b) and (d) of the same figures for a backward and a forward angle, with the slow (intermediate-velocity) source component represented with a dotted (dashed) line. The parameters extracted from the fit, multiplicity, and temperature of the slow source and multiplicity, temperature, and velocity of the intermediate-velocity source are reported in Tables II and III for protons and $\alpha$ particles, respectively. The quoted uncertainties correspond to the change in a parameter that increases $\chi^{2}$ by 1 , with all the other parameters fixed at their optimum values.

To evaluate the average energy taken away by preequilibrium neutrons, not detected in the present experiment, we assumed that their energy spectra were very similar to the proton ones, apart from the Coulomb barrier. Then the average kinetic energy of a preequilibrium neutron was taken to be that of a preequilibrium proton minus the Coulomb barrier while the preequilibrium neutron multiplicity was deduced by that of preequilibrium protons multiplied with the $N / Z$ ratio of the compound nucleus. The adopted preequilibrium neutron multiplicity is in agreement within errors with neutron emission studies performed at similar center-of-mass incident energy above the Coulomb barrier [31].

The values of average kinetic energy, binding energy, and energy lost for each pre-equilibrium particle are given for the ${ }^{40} \mathrm{Ca}+{ }^{152} \mathrm{Sm}$ and ${ }^{48} \mathrm{Ca}+{ }^{144} \mathrm{Sm}$ reactions in Tables IV and $\mathrm{V}$, respectively. The average excitation energy of the composite system after pre-equilibrium particle emission $E^{*}=E_{\text {c.m. }}+Q_{g g}-E_{\text {lost }}$, with $E_{\text {c.m. }}$ the energy available in the center-of-mass reference frame, $Q_{g g}$ the reaction ground state $Q$ value, and $E_{\text {lost }}$ the total energy lost, was deduced to be $E^{*}=(219.6 \pm 6.8) \mathrm{MeV}$ for the ${ }^{40} \mathrm{Ca}+{ }^{152} \mathrm{Sm}$ and $E^{*}=(220.5 \pm 6.6) \mathrm{MeV}$ for the ${ }^{48} \mathrm{Ca}+{ }^{144} \mathrm{Sm}$ reaction (see Table I). The average total mass and average total charge lost in each reaction were found to be $\Delta A=3.2 \pm 0.6$ and $\Delta Z=1.5 \pm 0.2$ for the ${ }^{40} \mathrm{Ca}+{ }^{152} \mathrm{Sm}$ system and $\Delta A=$ $3.4 \pm 0.5$ and $\Delta Z=1.6 \pm 0.2$ for the ${ }^{48} \mathrm{Ca}+{ }^{144} \mathrm{Sm}$ one. We considered, thus, that two units of charge and three units of mass were carried away from the initial composite system, leading to the ${ }^{189} \mathrm{Hg}$ nucleus in both reactions (see Table I). The average total momentum removed from the system was obtained by taking the product of the preequilibrium particle momentum with its multiplicity and then summing over particle types. This allowed us to derive the linear momentum transfer along the beam direction that was $\sim 97 \%$ in both reactions.

Summarizing the above results we deduce that, on average, the same compound system ${ }^{189} \mathrm{Hg}$ with the same excitation energy was formed, giving us confidence that any difference between the $\gamma$-ray spectra and $\gamma$-ray angular distributions of the two reactions is an entrance channel effect.

\section{B. $\gamma$-ray spectra}

Before a study of the $\gamma$-ray spectra can be performed, the incoherent bremsstrahlung component considered to originate primarily in neutron-proton $(n p)$ collisions and dominant for $E_{\gamma}>30 \mathrm{MeV}$, must be evaluated and subtracted from the data. An equal bremsstrahlung component is expected for the ${ }^{40,48} \mathrm{Ca}+{ }^{152,144} \mathrm{Sm}$ reactions because of their very similar beam energy and size of the reaction partners and of the same temperature of the composite system $[32,33]$. This is confirmed by the data of the two reactions, which are equal within errors for $E_{\gamma}>20 \mathrm{MeV}$.

The $n p$ bremsstrahlung component was deduced by fitting simultaneously the center-of-mass $\gamma$-ray multiplicity spectra of the two reactions at different polar angles in the energy range $30 \mathrm{MeV}<E_{\gamma}<40 \mathrm{MeV}$. The fit was performed assuming an exponentially decreasing behavior in the $n n$ center-of-mass system of the type

$$
\left(\frac{d^{2} M}{d \Omega d E_{\gamma}}\right)=M_{0} \exp \left(-\frac{E_{\gamma}}{E_{0}}\right)
$$

and an emitting source moving with $0.5 v_{\text {beam }}$ [34]. The coefficient $M_{0}$ and the inverse slope $E_{0}$ were obtained from a best fit to the data. The inverse slope was found to be $E_{0}=(7.6 \pm 2.2) \mathrm{MeV}$. In Fig. 5 we display the high-energy portion of the center-of-mass $\gamma$-ray multiplicity spectra of the two reactions (obtained with detectors centered at polar angles from $51.5^{\circ}$ to $128.5^{\circ}$ ), in coincidence with the evaporation residues along with the result of the fit. It is worth noting that the bremsstrahlung component at the present incident energy is small in the energy range of interest: it accounts for $13 \%$ of the total yield at $E_{\gamma}=20 \mathrm{MeV}$ and for $1.3 \%$ at $E_{\gamma}=10 \mathrm{MeV}$.

\section{Charge-symmetric reaction: Statistical GDR}

The bremsstrahlung-subtracted $\gamma$-ray spectrum of the nearly charge-symmetric reaction, ${ }^{48} \mathrm{Ca}+{ }^{144} \mathrm{Sm}$, is expected

TABLE III. As in Table II for $\alpha$ particles.

\begin{tabular}{lcccccrr}
\hline \hline Reaction & $E_{c}(\mathrm{MeV})$ & $v_{\mathrm{sl}}(\mathrm{cm} / \mathrm{ns})$ & $M_{\mathrm{sl}}$ & $T_{\mathrm{sl}}(\mathrm{MeV})$ & $v_{\text {int }}(\mathrm{cm} / \mathrm{ns})$ & $M_{\text {int }}$ & $T_{\text {int }}(\mathrm{MeV})$ \\
\hline${ }^{40} \mathrm{Ca}+{ }^{152} \mathrm{Sm}$ & 14 & 0.937 & $2.070 \pm 0.100$ & $3.870 \pm 0.052$ & $1.607 \pm 0.126$ & $0.343 \pm 0.075$ & $6.261 \pm 0.243$ \\
${ }^{48} \mathrm{Ca}+{ }^{144} \mathrm{Sm}$ & 14 & 1.028 & $2.568 \pm 0.099$ & $3.595 \pm 0.047$ & $1.860 \pm 0.088$ & $0.443 \pm 0.059$ & $6.502 \pm 0.160$ \\
\hline \hline
\end{tabular}


TABLE IV. Average kinetic energy $E_{k}$, binding energy $E_{\text {bind }}$, preequilibrium particle multiplicity $M_{\text {int }}$, and average energy lost $E_{\text {lost }}$ for the ${ }^{40} \mathrm{Ca}+{ }^{152} \mathrm{Sm}$ reaction.

\begin{tabular}{lrcrr}
\hline \hline Particle & \multicolumn{1}{c}{$E_{k}(\mathrm{MeV})$} & $E_{\text {bind }}(\mathrm{MeV})$ & $M_{\text {int }}$ & $E_{\text {lost }}=M_{\text {int }}\left[E_{k}+E_{\text {bind }}\right](\mathrm{MeV})$ \\
\hline Proton & $13.71 \pm 0.13$ & 3.56 & $0.786 \pm 0.060$ & $13.57 \pm 0.95$ \\
Neutron & $7.71 \pm 0.13$ & 10.56 & $1.055 \pm 0.250$ & $19.27 \pm 4.53$ \\
$\alpha$ & $23.39 \pm 0.36$ & -5.00 & $0.343 \pm 0.075$ & $6.31 \pm 1.28$ \\
\hline \hline
\end{tabular}

to be adequately described by the statistical $\gamma$ spectrum of the compound nucleus decay, calculated with the code CASCADE. This holds because the DD $\gamma$ radiation in this channel is expected to be negligible, its initial dipole moment being very low. From the comparison of the ${ }^{48} \mathrm{Ca}+{ }^{144} \mathrm{Sm}$ data with CASCADE calculations, new information can be obtained on the statistical GDR in the highly excited ${ }^{189} \mathrm{Hg}$ compound nucleus and in its evaporation daughters.

Before presenting our results, in the following we summarize the existing data on the GDR properties for neighboring heavy nuclei. The ground-state GDR centroid energy, width, and strength for a nucleus of mass $A \approx 190$ are expected to be: $E_{\mathrm{GDR}, \mathrm{gs}} \approx 14 \mathrm{MeV}, \Gamma_{\mathrm{GDR}, \mathrm{gs}}=5-6 \mathrm{MeV}$, and $S_{\mathrm{GDR}, \mathrm{gs}} \gtrsim 1$ in units of the $E 1$ energy-weighted sum rule (EWSR) [35,36]. The study of the GDR $\gamma$ decay from excited nuclei in this mass region was limited so far to lower excitation energies than presented here, and it was mainly performed with the aim to investigate superdeformed nuclear shapes predicted by calculations. These calculations, done for a ${ }^{196} \mathrm{~Pb}$ nucleus, spherical in its ground state, predicted a prolate superdeformed minimum at $T=0 \mathrm{MeV}$ and $J=30 \hbar$ with deformation parameter $\beta=0.6$. The prolate minimum washed up at $T>1 \mathrm{MeV}$ where a phase transition to a noncollective oblate shape was expected to occur (see Refs. [37,38] and references therein). Heavy-ion fusion experiments probed the nuclear shape of ${ }^{200} \mathrm{~Pb}$ isotopes at temperatures extending up to $1.5 \mathrm{MeV}$ and spins extending up to $44 \hbar$ in evaporation $[39,40]$ and $50 \hbar$ in fission events [38]. The above temperature refers to that of nuclear states upon which the GDR was built averaging over the particle decay steps for which $\gamma$ decay with $E_{\gamma}>10 \mathrm{MeV}$ was significant. These works, in agreement with theoretical predictions, suggested a spherical-to-prolate nuclear shape transition at a spin value of $14 \hbar-18 \hbar$ that persisted at a temperature of $1.3 \mathrm{MeV}$. In fact, for temperatures up to $T \sim 1.3 \mathrm{MeV}$, two Lorentzian components and a prolate deformation with $\beta \sim 0.3$, were necessary in the CASCADE calculation to reproduce the GDR $\gamma$ strength function while at $T=1.5 \mathrm{MeV}$, a single Lorentzian component, with $E_{\mathrm{GDR}}=$ 13.8 $\mathrm{MeV}$ and $\Gamma_{\mathrm{GDR}}=9 \mathrm{MeV}$, was found to be adequate [39].

Another fusion experiment was performed to investigate the shape of ${ }^{197} \mathrm{Tl}$ isotopes formed at excitation energies up to $71.6 \mathrm{MeV}$ and maximum value of the compound nucleus spin distribution equal to $49 \hbar$ [41]. The GDR centroid energy needed to explain the $\gamma$ strength functions was found to be $E_{\mathrm{GDR}}=13.5 \mathrm{MeV}$, compatible with the ground-state value, the width of the distribution $\Gamma_{\mathrm{GDR}} \sim 8 \mathrm{MeV}$, while the mean nuclear deformations were found to be $\beta \sim 0.25$. In that work, no definite conclusions were drawn on the type of the nuclear deformation.

Besides fusion experiments between heavy ions, an $\alpha$ scattering experiment on a ${ }^{208} \mathrm{~Pb}$ target was employed to study the GDR $\gamma$ decay from the excited ${ }^{208} \mathrm{~Pb}$ nucleus. The $\alpha$ scattering method populated highly excited nuclear states of the target nucleus at low angular momenta, making it possible to isolate the evolution of the GDR properties with temperature [42]. It was found that the GDR width increases continuously with the temperature in a range varying from 1 to $1.6 \mathrm{MeV}$, where it takes a value of $8 \mathrm{MeV}$, much larger than the ground-state value of $4 \mathrm{MeV}$ (for the reevaluation of the temperatures of the nuclear states on which the GDR was built see Ref. [43]). The width increase was ascribed to the nuclear shape thermal fluctuations.

In the present work, we evaluated the statistical $\gamma$-ray emission in the nearly charge-symmetric channel by means of the CASCADE code for a ${ }^{189} \mathrm{Hg}$ compound nucleus formed at excitation energy $E^{*}=220.5 \mathrm{MeV}$. The spin distribution of evaporation and fission events was derived from a PACE2 calculation performed for the above-mentioned compound nucleus and by using a level density parameter $a=A / 9.5 \mathrm{MeV}^{-1}$. The evaporation cross section was found to be $132 \mathrm{mb}$, corresponding to a critical spin $J_{\text {critevap }}=45 \hbar$ in the sharp cutoff approximation. The statistical fission cross section was found to be $242 \mathrm{mb}$ and accounts for the remaining part of the compound nucleus formation cross section up to the $J_{B_{f}=0}$ value where the fission barrier vanishes. The

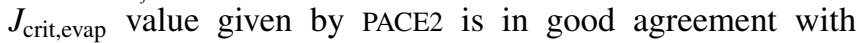
estimations of the critical angular momentum for evaporation residue products owing to fission competition for a compound nucleus with charge $Z \sim 80$ [44] and with the evaporation data of the neighboring systems ${ }^{48} \mathrm{Ca}+{ }^{154} \mathrm{Sm}[45],{ }^{30} \mathrm{Si}+{ }^{170} \mathrm{Er}$, and ${ }^{19} \mathrm{~F}+{ }^{181} \mathrm{Ta}$ [46]. In the CASCADE calculation we used a triangular spin distribution with a critical value equal to

TABLE V. Same as in Table IV for the ${ }^{48} \mathrm{Ca}+{ }^{144} \mathrm{Sm}$ reaction.

\begin{tabular}{lrcrr}
\hline \hline Particle & \multicolumn{1}{c}{$E_{k}(\mathrm{MeV})$} & $E_{\text {bind }}(\mathrm{MeV})$ & $M_{\text {int }}$ & $E_{\text {lost }}=M_{\text {int }}\left[E_{k}+E_{\text {bind }}\right](\mathrm{MeV})$ \\
\hline Proton & $13.51 \pm 0.16$ & 3.56 & $0.693 \pm 0.062$ & $11.83 \pm 0.98$ \\
Neutron & $7.51 \pm 0.16$ & 10.56 & $0.930 \pm 0.250$ & $16.81 \pm 4.48$ \\
$\alpha$ & $23.75 \pm 0.24$ & -5.00 & $0.443 \pm 0.059$ & $8.31 \pm 1.18$ \\
\hline \hline
\end{tabular}




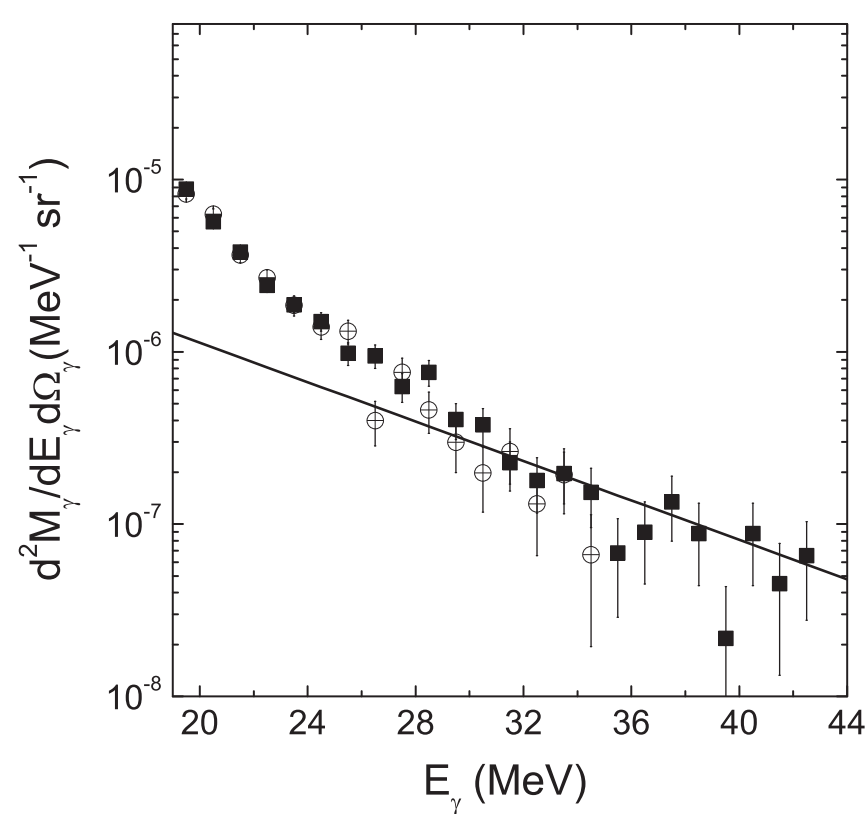

FIG. 5. High-energy range of the center-of-mass $\gamma$-ray multiplicity spectra, obtained with detectors centered at polar angles from $51.5^{\circ}$ to $128.5^{\circ}$, in coincidence with the evaporation residues for the ${ }^{40} \mathrm{Ca}+{ }^{152} \mathrm{Sm}$ (solid squares) and the ${ }^{48} \mathrm{Ca}+{ }^{144} \mathrm{Sm}$ (open circles) reaction. The solid line represents the bremsstrahlung component determined from the simultaneous fit of the data, as described in the text.

$45 \hbar$ and a diffusivity parameter $2 \hbar$. The above distribution results in an average spin of the compound nucleus for evaporation events $\bar{J}_{\mathrm{CN} \text {,evap }}=31 \hbar$ and a corresponding average temperature $\bar{T}_{\mathrm{CN} \text {,evap }}=3.2 \mathrm{MeV}$, calculated from the relation $\left(E^{*}-E_{\mathrm{rot}}\right)=a \bar{T}_{\mathrm{CN} \text {,evap }}^{2}, E_{\mathrm{rot}}$ being the rotational energy for $\bar{J}_{\mathrm{CN} \text {,evap }}$ and a nuclear level density parameter $a=A / 9.5 \mathrm{MeV}^{-1}$.

It is well known that $a$ is of primary importance for the statistical $\gamma$ spectrum. Different measurements have shown that (a) $a$ decreases with increasing excitation energy from a value $A / 8-A / 9 \mathrm{MeV}^{-1}$ to a value $\sim A / 13 \mathrm{MeV}^{-1}$ [47] and (b) the use of an energy-independent level density parameter lower than $A / 8 \mathrm{MeV}^{-1}$ or the use of a modest energy dependence makes it possible to explain equally well charged-particle evaporation spectra relative to compound nuclei with various masses [48-50]. The same holds also for statistical $\gamma$-ray spectra that have been successfully analyzed by using both approaches. Although many works have been dedicated to the study of the level density parameter, uncertainties still remain concerning it. Thus, in the following, we consider different prescriptions in the CASCADE calculations.

(i) The CASCADE approach proposed by Puhlhofer [22], by using a constant level density parameter $a=A / 9.5 \mathrm{MeV}^{-1}$ in the "high-energy" region, $E^{*} \gtrsim 20 \mathrm{MeV}$. To obtain the sensitivity of our results, a second calculation was done with $a=A / 11 \mathrm{MeV}^{-1}$ because this choice produced the best description of both proton and $\alpha$-particle spectra for a ${ }^{193} \mathrm{Tl}$ compound nucleus when using an energy-independent level density parameter approach [48]. (ii) The semiempirical level density prescription of Reisdorf [51] with the ansatz of Ignatyuk [52] that reflects the nuclear shell structure at low excitation energy and its smooth melting away at high excitation energy. In this approach, $a$ has an asymptotic value $A / 9.4 \mathrm{MeV}^{-1}$ for a compound nucleus with $A=189$.

(iii) The prescription proposed by Charity [50] that considers (a) a level density parameter, $\tilde{a}_{\text {eff }}$, depending on excitation energy and mass and (b) an additional slow energy dependence like that given by the Ignatyuk expression [52] for the disappearance of the shell effects with the inclusion of a term depending on the nuclear spin. This approach was already used in the literature to reproduce charged particle [50] and $\gamma$-ray spectra [53]. For a mass $A=$ $189, \tilde{a}_{\text {eff }}$ varies from $A / 7.3 \mathrm{MeV}^{-1}$ at low temperature to $A / 9.9 \mathrm{MeV}^{-1}$ at $T=3.2 \mathrm{MeV}$ (according to Equation (15) of [50]).

(iv) The parametrization suggested by Ormand et al. in Ref. [54], who proposed a level density parameter, renormalized by small amplitude (quantal) fluctuations of the nuclear surface. In this approach, $a$ varies from $A / 8.6 \mathrm{MeV}^{-1}$ at low temperature to $A / 10.6 \mathrm{MeV}^{-1}$ at $T=3.2 \mathrm{MeV}$. In addition, shell effects on the level density at low energy were taken into account by using the Ignatyuk ansatz [52].

For the CASCADE calculation using the prescriptions (ii), (iii), and (iv), we modified the level density part of the code [22] according to Ref. [55] and we calculated the thermal energy and the shell and pairing corrections to the liquid drop mass as described in Ref. [55]. Moreover, in all approaches, the Myers droplet model mass formula [56] with the inclusion of the Wigner term was employed for obtaining the binding energy of the spherical liquid drop without shell and even-odd correction. Finally, in all CASCADE calculations the GDR strength function was taken to be a single Lorentzian curve throughout the decay cascade.

The resulting statistical $\gamma$ spectra obtained with the previously described prescriptions of the level density parameter are displayed in Figs. 6 and 7, together with the data. In the left-hand side of these figures we report the center-of-mass bremsstrahlung-subtracted $\gamma$-ray multiplicity spectrum of the ${ }^{48} \mathrm{Ca}+{ }^{144} \mathrm{Sm}$ reaction (symbols), taken with detectors at polar angles from $68.1^{\circ}$ to $128.5^{\circ}$, together with the theoretical spectrum (line) obtained with the code CASCADE and folded with the response function to $\gamma$ rays of the considered $\mathrm{BaF}_{2}$ rings [57]. The error bars of the data include both the statistical uncertainties and the errors owing to the subtraction of the bremsstrahlung radiation. To approximately remove the exponential behavior of the spectrum reflecting the nuclear level density energy dependence and to compare on a linear scale the data with the fitted $E 1$ absorption cross section, $\sigma_{\text {abs }}\left(E_{\gamma}\right)$, we divided both data and theoretical $\gamma$ spectrum by the theoretical $\gamma$ spectrum and multiplied by the $E 1$ absorption cross section used in the CASCADE calculation. The resulting linearized spectra are shown in the right-hand side panels of the figures. In Fig. 6 we present calculations by using the Puhlhofer approach with $a=A / 9.5 \mathrm{MeV}^{-1}$ (top) and $a=A / 11 \mathrm{MeV}^{-1}$ (bottom). In Fig. 7 we display the calculations by using the Reisdorf approach (top), the Charity approach (middle), and the Ormand approach (bottom). 

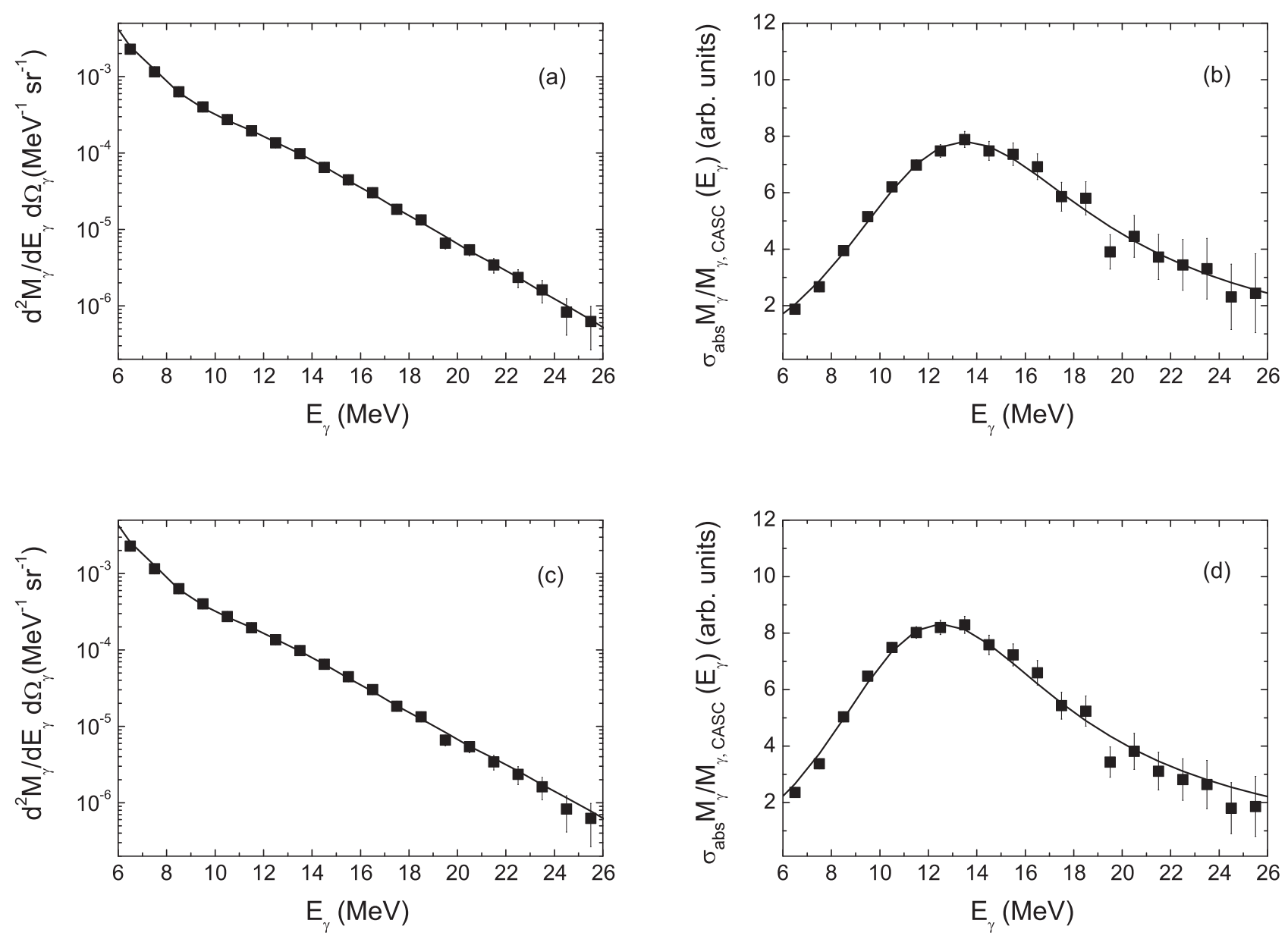

FIG. 6. (a) Center-of-mass bremsstrahlung-subtracted $\gamma$-ray multiplicity spectrum of the charge-symmetric reaction ${ }^{48} \mathrm{Ca}+{ }^{144} \mathrm{Sm}$, obtained with detectors centered at polar angles from $68.1^{\circ}$ to $128.5^{\circ}$, in coincidence with evaporation residues (symbols). The line represents the statistical $\gamma$-ray spectrum calculated with the code CASCADE and folded with the response function of the considered $\mathrm{BaF}_{2}$ rings. The CASCADE calculation was done by using the Puhlhofer prescription for the level density parameter and with $a=A / 9.5 \mathrm{MeV}^{-1}$ in the "high-energy" region. (b) The linearized plot obtained by dividing the spectra shown in panel (a) by the CASCADE spectrum and multiplied by the $E 1$ absorption cross section, $\sigma_{\mathrm{abs}}\left(E_{\gamma}\right)$, used in the CASCADE calculation. The parameters of $\sigma_{\mathrm{abs}}\left(E_{\gamma}\right)$ are presented in the first row of Table VI. Panels (c) and (d) are as (a) and (b), respectively, obtained by using $a=A / 11 \mathrm{MeV}^{-1}$ in the "high-energy" region. The corresponding parameters of $\sigma_{\mathrm{abs}}\left(E_{\gamma}\right)$ are presented in the second row of Table VI.

The GDR parameters, corresponding to the $\sigma_{\mathrm{abs}}\left(E_{\gamma}\right)$, that were used to reproduce our $\gamma$ spectra in the different prescriptions of the level density parameter are presented in Table VI. An uncertainty of $10 \hbar$ in the $J_{\text {critevap }}$ value and $7 \mathrm{MeV}$ in the compound nucleus excitation energy does not affect our results on the GDR parameters by more than the quoted errors.

By examining the different cases and looking at Figs. 6 and 7 , we observe that all the considered prescriptions describe reasonably well the data, except for the Ormand prescription that fails essentially in the high-energy region. This prescription, used successfully in previous works $[18,58]$ to reproduce $\gamma$-ray spectra originating from compound nuclei in the $A \sim 130$ mass region, seems to give a too-rapidly varying level density parameter, overpredicting thus the experimental spectra at high energies. The best description of the data in the whole energy range of interest is reached by using the Puhlhofer approach with $a=A / 9.5 \mathrm{MeV}^{-1}$ or the Reisdorf approach that results in a similar value of the level density, nearly constant throughout the whole decay. With the $a=A / 11 \mathrm{MeV}^{-1}$ choice we obtain a centroid energy that is $11 \%$ lower than that of the ground-state value, indicating probably that this choice is not the most appropriate in our case. In the Charity approach, we present here the calculation including the spin term in the melting out of the shell effects (see Eq. (12) of [50]); however, the exclusion of such a term does not influence much the results. If the spin term is not included, we extract the following parameters: $E_{\mathrm{GDR}}=14.0 \mathrm{MeV}, \Gamma_{\mathrm{GDR}}=11.0 \mathrm{MeV}$, and $S_{\mathrm{GDR}}=1.25 E 1$ EWSR. As a final comment, we notice that in both the Charity and the Ormand approaches where an energy-dependent $a$ is used, the extracted GDR width is lower than by using a constant (or nearly constant) $a$ in the decay cascade.

Even if we obtain the best results with the $a=$ A/9.5 $\mathrm{MeV}^{-1}$ choice of the Puhlhofer approach or with the similar $a$ value of the Reisdorf approach, we do not have strong physical arguments to choose the most appropriate prescription. Then we consider in the following the average of 

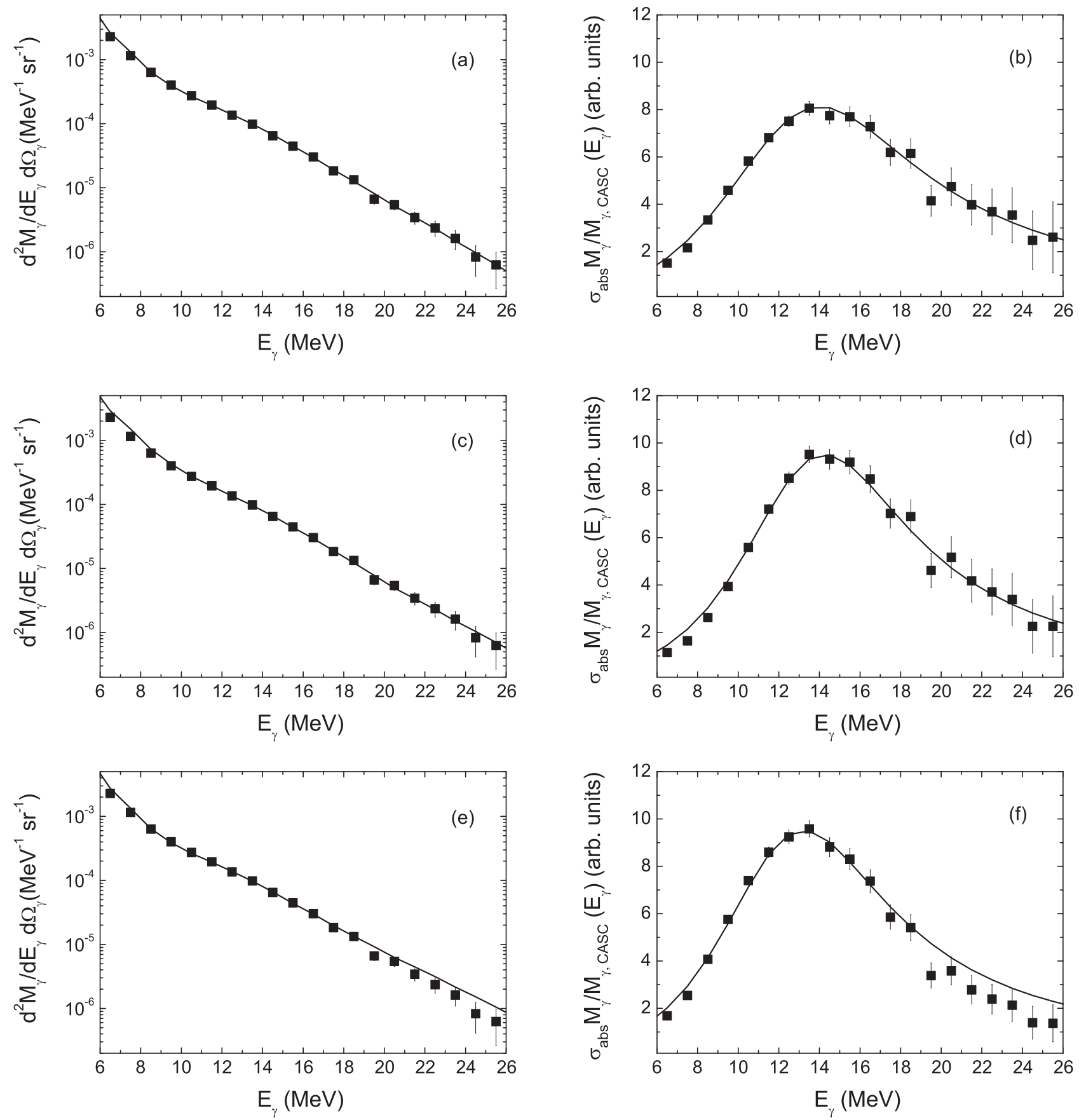

FIG. 7. (From top to bottom) The same as in Fig. 6 with the parameters of $\sigma_{\mathrm{abs}}\left(E_{\gamma}\right)$ presented in the third, fourth, and fifth rows of Table VI. Panels (a) and (b) correspond to the Reisdorf approach, (c) and (d) to the Charity approach, and (e) and (f) to the Ormand approach.

the extracted GDR parameters from all approaches considering them on the same foot. The average is shown in the last row of Table VI.

To compare our results with previous ones on the same basis, we evaluated the average values of mass $\bar{A}_{f}$, charge $\bar{Z}_{f}$, temperature $\bar{T}_{f}$, and spin $\bar{J}_{f}$ of the nuclei in the decay cascade following GDR $\gamma$-ray emission. These quantities are displayed in Table VI. The average temperature of the nuclear states upon which the GDR is built was obtained from the relation $\bar{E}_{f}=a \bar{T}_{f}^{2}$, with $\bar{E}_{f}=\bar{E}_{\mathrm{in}}-E_{\mathrm{rot}}-E_{\mathrm{GDR}}$ being
$\bar{E}_{\text {in }}$ the average nuclear excitation energy minus the energy lost by particle evaporation and $E_{\text {rot }}$ the rotational energy calculated for $\bar{J}_{f}$. The level density parameter was taken to be $a=\bar{A}_{f} / 9.5 \mathrm{MeV}^{-1}$. The weighted average of the above quantities was performed over all nuclei in the decay cascade emitting $\gamma$ rays with $E_{\gamma}=E_{\mathrm{GDR}}$, using the code CASCADE with GDR parameters extracted in the Puhlhofer approach (see first row of Table VI). An uncertainty of $\Delta k= \pm 1.5 \mathrm{MeV}$, where $k=A / a$, introduces an error of $\pm 0.20 \mathrm{MeV}$ in the determination of the $\bar{T}_{f}$ value. 
TABLE VI. GDR parameters extracted with different prescriptions of the level density. The associated errors are $\pm 0.3 \mathrm{MeV}$ for the centroid energy, $\pm 0.5 \mathrm{MeV}$ for the width, and \pm 0.05 of the $E 1$ EWSR for the strength. The average result of the above GDR parameters is shown in the last row. Average values of mass $\bar{A}_{f}$, charge $\bar{Z}_{f}$, temperature $\bar{T}_{f}$, and spin $\bar{J}_{f}$ of the nuclei in the decay cascade following emission of GDR $\gamma$ rays, obtained with a CASCADE calculation and $a=A / 9.5 \mathrm{MeV}^{-1}$ in the Puhlhofer approach (for details, see the text).

\begin{tabular}{lccccc}
\hline \hline $\begin{array}{l}E_{\mathrm{GDR}} \\
(\mathrm{MeV})\end{array}$ & $\begin{array}{c}\Gamma_{\mathrm{GDR}} \\
(\mathrm{MeV})\end{array}$ & $\begin{array}{c}S_{\mathrm{GDR}} \\
(E 1 \mathrm{EWSR})\end{array}$ & Level density parameter & $\bar{A}_{f}$ & $\begin{array}{c}\bar{Z}_{f} \\
\bar{J}_{f} \\
(\hbar)\end{array}$ \\
\hline 13.5 & 12.8 & 1.01 & Puhlhofer $\left(A / 9.5 \mathrm{MeV}^{-1}\right)$ & 181 & \\
12.5 & 12.0 & 1.04 & Puhlhofer $\left(A / 11 \mathrm{MeV}^{-1}\right)$ & & \\
$(\mathrm{MeV})$ & & \\
14.0 & 12.3 & 1.03 & Reisdorf & \\
14.25 & 10.5 & 1.08 & Charity & \\
13.2 & 10.5 & 0.96 & Ormand & \\
13.5 & 11.6 & 1.02 & Average GDR parameters & & \\
\hline \hline
\end{tabular}

From our results on the average GDR parameters we note the following.

(i) The centroid energy, $E_{\mathrm{GDR}}=13.5 \mathrm{MeV}$, is in agreement with the ground-state value $E_{\mathrm{GDR}, \mathrm{gs}}$ within $4 \%$ and consistent with previous experimental results and theoretical predictions [59-62].

(ii) The width, $\Gamma_{\mathrm{GDR}}=11.6 \mathrm{MeV}$, increases with the nuclear temperature and/or the excitation energy, if compared with the results obtained for Pb isotopes: $9 \mathrm{MeV}$ at $T=1.5 \mathrm{MeV}$ and similar spin distribution [39] and $8 \mathrm{MeV}$ at $T \sim 1.6 \mathrm{MeV}$ and much lower average spin [42]. The extracted width is, moreover, in good agreement with the $11.2 \mathrm{MeV}$ value derived by means of the phenomenological relation (4) of Ref. [43], which describes the width behavior as a function of temperature, spin, and mass in the framework of the thermal fluctuation model. The following values were used in the phenomenological relation: $\Gamma_{\mathrm{GDR}, \mathrm{gs}}=$ $5.5 \mathrm{MeV}, \bar{T}_{f}=2.33 \mathrm{MeV}, \bar{J}_{f}=28 \hbar$. The width increase suggests an increase of the nuclear deformation. By making use of the universal linear relation between the GDR width and the mean nuclear quadrupole deformation derived in the framework of the thermal fluctuation model [63], a mean deformation parameter of $\beta \sim 0.4-0.5$ is obtained from our data. The lower and higher $\beta$ value corresponds, respectively, to the lower and higher GDR width shown in Table VI.

(iii) The strength exhausted by the GDR was found to be $S_{\mathrm{GDR}} \approx 1 E 1 \mathrm{EWSR}$, in agreement with the ground-state value for nuclei in this mass region. Thus, no $\gamma$-ray emission cutoff in the CASCADE calculation was needed to reproduce the data in the GDR energy region. Such GDR $\gamma$-ray suppression was observed for energies above $E^{*} \sim 2 \mathrm{MeV} /$ nucleon for compound nuclei with mass $A \sim 130[18,58]$, $\sim 2.2 \mathrm{MeV} /$ nucleon for $A \sim 110[58,64,65]$, and $\sim 5 \mathrm{MeV} /$ nucleon for lighter nuclei $A=60-70$ [12], denoting a mass dependence of the limiting excitation energy for the collective motion. This dependence is very similar to the mass dependence of the limiting temperature that nuclei can sustain before undergoing multifragmentation, as observed from the analysis of the nuclear caloric curves for nuclei in different mass regions [47]. In that work, the excitation energy per nucleon at which a limiting temperature was reached for masses $180 \leqslant A \leqslant 240$ was found to be $2-2.5 \mathrm{MeV} /$ nucleon. As in the present work, the compound nucleus was formed at $E^{*}=1.2 \mathrm{MeV} /$ nucleon, our result evidences the persistence of collective motion at the considered excitation energy. Assuming that there could be a link between the disappearance of the GDR collective motion and a liquid-gas phase transition, this result is in agreement with what we would expect from the nuclear caloric curve study.

\section{Charge-asymmetric reaction: Preequilibrium $\gamma$ emission}

It has been demonstrated in the previous sections that the only difference between the studied entrance channels concerns their charge asymmetry and that the associated bremsstrahlung-subtracted $\gamma$-ray spectra can be safely compared with each other. Because the same compound nucleus was formed at identical conditions, if one considers only the statistical decay of the compound nucleus, extensively presented in Sec. III B 1, the spectra of the two channels should be identical.

In panel (a) of Fig. 8 we display the center-of-mass bremsstrahlung-subtracted $\gamma$-ray multiplicity spectra of the two reactions, obtained with detectors centered at polar angles from $51.5^{\circ}$ to $128.5^{\circ}$, in coincidence with the evaporation residues. The solid (dashed) line represents the charge-asymmetric ${ }^{40} \mathrm{Ca}+{ }^{152} \mathrm{Sm}$ (nearly charge-symmetric ${ }^{48} \mathrm{Ca}+{ }^{144} \mathrm{Sm}$ ) reaction. The symbols in panel (b) correspond to their difference. In panel (c) is presented the percent difference between the data, i.e., the ratio of the difference and the spectrum of the ${ }^{48} \mathrm{Ca}+{ }^{144} \mathrm{Sm}$ reaction. The data show an excess of $\gamma$ rays in the range $E_{\gamma}=8-15 \mathrm{MeV}$ in the charge-asymmetric channel. This excess can only be related to the DD excitation in the composite system of the ${ }^{40} \mathrm{Ca}+{ }^{152} \mathrm{Sm}$ reaction because of its larger charge asymmetry. The DD $\gamma$ spectrum [symbols in panel (b)] can be reproduced well by means of a Lorentzian curve folded with the response function of the considered $\mathrm{BaF}_{2}$ rings [57] [solid line in panel (b)]. The extracted characteristics, centroid energy $E_{\mathrm{DD} \text {,exp }}$, and width $\Gamma_{\text {DD,exp }}$ are given in Table VII. 

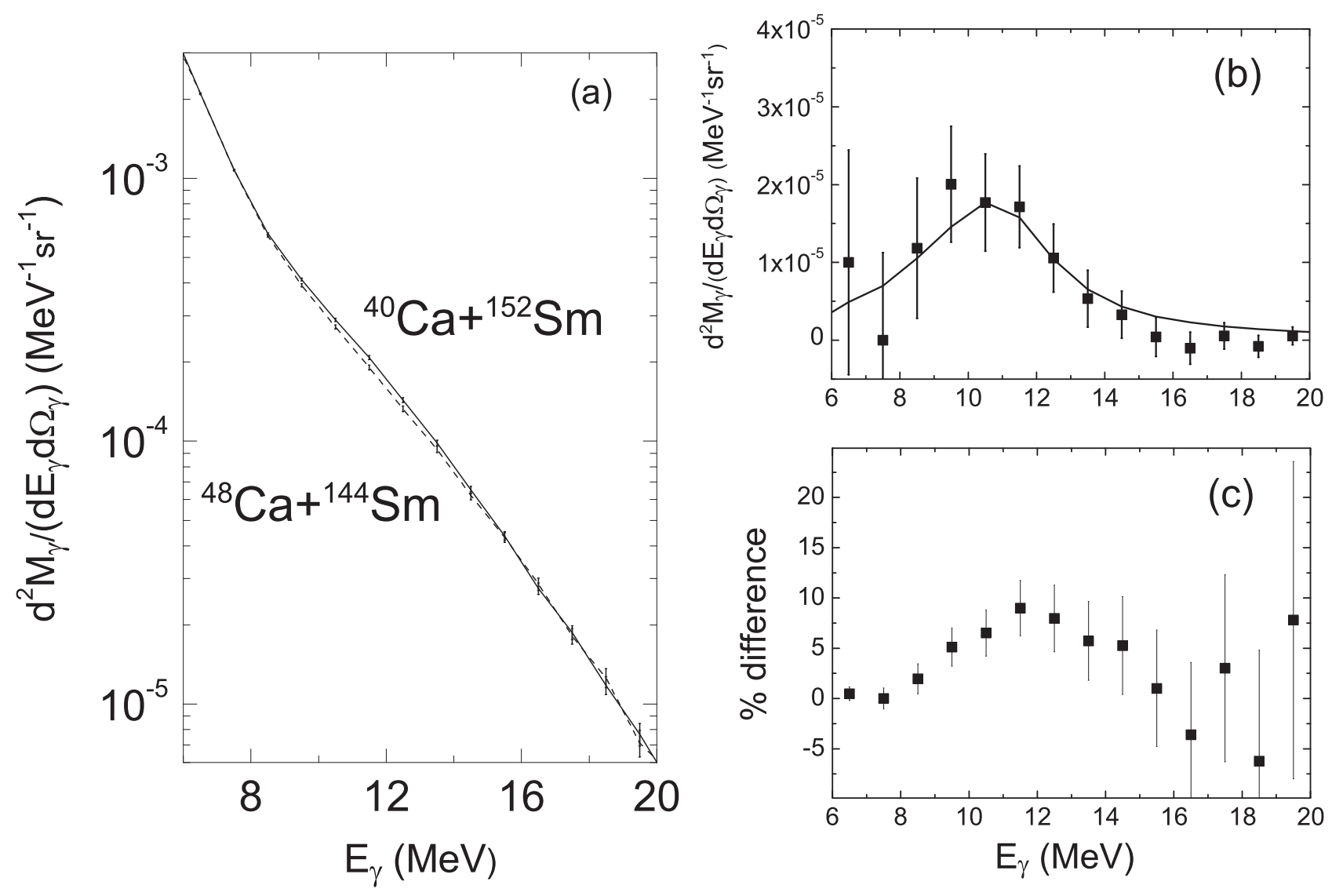

FIG. 8. (a) Center-of-mass bremsstrahlung-subtracted $\gamma$-ray multiplicity spectra, obtained with detectors at polar angles from $51.5^{\circ}$ to $128.5^{\circ}$, in coincidence with evaporation residues. The solid (dashed) line represents the charge-asymmetric ${ }^{40} \mathrm{Ca}+{ }^{152} \mathrm{Sm}$ (nearly chargesymmetric ${ }^{48} \mathrm{Ca}+{ }^{144} \mathrm{Sm}$ ) reaction. (b) The symbols correspond to the difference between the experimental spectra of panel (a), i.e., the DD $\gamma$-ray spectrum. The solid line is a Lorentzian curve folded with the response function of the considered $\mathrm{BaF}_{2}$ rings with characteristics described in the text and presented in Table VII. (c) Percent difference between the data, i.e., ratio of the difference shown in panel (b) and the spectrum of the ${ }^{48} \mathrm{Ca}+{ }^{144} \mathrm{Sm}$ reaction shown in panel (a).

Because the DD centroid energy reflects the emitting source deformation, it is interesting to compare it with the centroid energy of the ground-state GDR, $E_{\mathrm{GDR}, \mathrm{gs}}$. We notice that $E_{\mathrm{DD} \text {,exp }}$ is $3 \mathrm{MeV}$ lower than $E_{\mathrm{GDR}, \mathrm{gs}}$, indicating a high deformation of the emitting source during the DD $\gamma$ emission. This result is in excellent agreement with expectations for a dipole oscillation along the symmetry axis of a deformed dinucleus during the early moments of the reaction [3-10] and with previous experimental works on lighter systems $[10,13,15,17,18]$.

\section{3. $\gamma$-ray angular distributions}

The center-of-mass bremsstrahlung-subtracted $\gamma$-ray angular distribution with respect to the beam direction of the two reactions, corrected for the detection efficiency, was fitted with a Legendre polynomial expansion,

$$
M_{\gamma}\left(E_{\gamma}, \theta_{\gamma}\right)=M_{0}\left(E_{\gamma}\right)\left\{1+Q_{2} a_{2}\left(E_{\gamma}\right) P_{2}\left[\cos \left(\theta_{\gamma}\right)\right]\right\},
$$

where $a_{2}$ is the anisotropy coefficient and $Q_{2}$ is an attenuation factor for the finite $\gamma$-ray counter which, for the present

TABLE VII. The centroid energy $E_{\mathrm{DD} \text {,exp }}$ and width $\Gamma_{\mathrm{DD} \text {,exp }}$ of the Lorentzian curve that describes the experimental DD $\gamma$-ray spectrum shown in panel (b) of Fig. 8. Experimental energy- and angle-integrated DD yield $M_{\gamma, \mathrm{DD} \text {,exp }}$ corrected for the detection efficiency. The cited error on the $\mathrm{DD}$ yield includes uncertainty in integration over angle and $\mathrm{BaF}_{2}$ scintillator efficiency. A 7-MeV difference in the compound nucleus $E^{*}$ between the two reactions (see Table I) would introduce an additional error of $\pm 0.3 \times 10^{-3}$. The DD parameters obtained from BNV calculations with an asystiff EOS (in parentheses the values with an asysoft EOS) are shown in the following columns: centroid energy $E_{\mathrm{DD}, \mathrm{th}}$, width $\Gamma_{\mathrm{DD}, \mathrm{th}}$, and yield $M_{\gamma, \mathrm{DD} \text {,th }}$. The impact parameter window considered for the calculation of the theoretical DD parameters is taken from 0 up to the $b_{\max }$ value indicated in the last column.

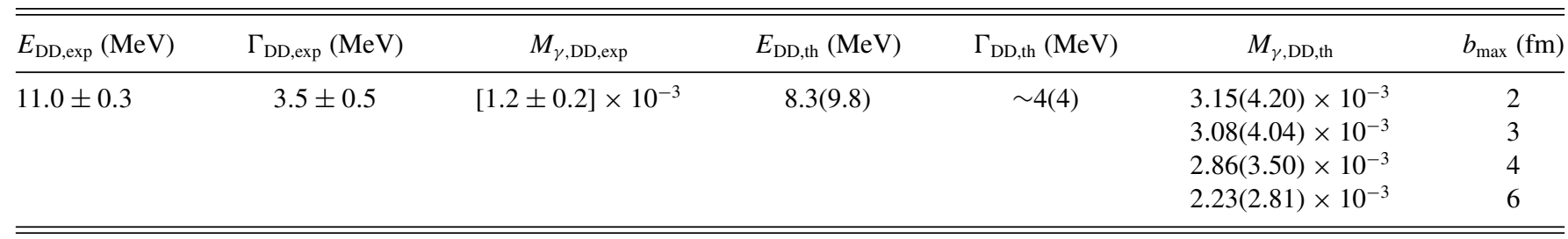


geometry, was found to be 0.98 [66]. The detection efficiency of the $\mathrm{BaF}_{2}$ ring centered at the polar angle $\theta_{\gamma}$ was deduced from its response function [57]. The coefficients $M_{0}\left(E_{\gamma}\right)$ and $a_{2}\left(E_{\gamma}\right)$ were obtained from a best fit of the data.

In panels (a) and (b) of Fig. 9 we show the energy dependence of the $a_{2}$ coefficient in bins of $1 \mathrm{MeV}$. For the nearly charge-symmetric system ${ }^{48} \mathrm{Ca}+{ }^{144} \mathrm{Sm}, a_{2}$ is expected to have an energy dependence characteristic of statistical GDR $\gamma$ decay from a deformed hot rotating nucleus. We recall here that we do not expect DD $\gamma$ radiation in this channel, the initial dipole moment being very low. In the case of an oblate shape rotating noncollectively, as should be in the present case, the two low-energy GDR components (along the two longer axes of the nuclear shape) should have a minimum $a_{2} \sim-0.25$ (stretched dipole transitions $\Delta J= \pm 1$ ), while the upper-energy GDR component (along the shorter axis) should have an $a_{2} \sim 0.5$ (unstretched dipole transition $\Delta J=0$ ). For a detailed discussion, see, for instance, Ref. [67]. However, the overlap of the split GDR components because of their finite width, the nuclear shape thermal fluctuations, and the fluctuations of the orientation of the angular frequency vector with respect to that of the density distribution, as predicted by the fluctuation theory of a heated rotating liquid drop [68], render the angular distribution of GDR $\gamma$ rays more complex and modify the value of the relative $a_{2}$. Measured $a_{2}$ were found to be smaller in absolute value than those described previously for statistical GDR $\gamma$ decay from excited nuclei, depending on the nuclear excitation energy and on the nuclear spin.

Our data confirm the above considerations. In the lowenergy side of the GDR $\left(E_{\gamma}<13 \mathrm{MeV}\right)$, the chargesymmetric reaction data in panel (b) of Fig. 9 show a minimum $a_{2}=-0.12$, while on the high-energy side, the $a_{2}$ coefficient has a slightly positive value with large error bars. The lowenergy part $a_{2}$ is significantly attenuated (in absolute value) with respect to the $a_{2} \sim-0.25$ value expected for a stretched dipole transition. As explained previously, this attenuation is ascribed to the orientation fluctuations of the compound nucleus angular frequency vector with respect to that of the density distribution. The attenuation becomes more important at low rotational frequencies, as in the present case, where $\omega=0.3-0.35 \mathrm{MeV}$ for the involved heavy nuclei that emit GDR $\gamma$ radiation. It has to be noticed that, while orientation fluctuations of the angular frequency vector are very important to describe the GDR $\gamma$-ray angular distribution, they do not influence the GDR $\gamma$ strength function that depends mainly on the large amplitude thermal fluctuations of the nuclear shape [68]. Our result on the statistical GDR $a_{2}$ coefficient, though at higher excitation energy, is in agreement with that obtained for $\mathrm{Tl}$ isotopes at an excitation energy of $60 \mathrm{MeV}$ and a compound nucleus average spin of $24 \hbar$ [41], where large orientation fluctuations of the spin vector were needed to explain the observed near isotropy across the GDR region. Furthermore, it is compatible with the experimental findings of Ref. [69] for a ${ }^{176} \mathrm{~W}$ nucleus formed at $T=1.4 \mathrm{MeV}$ and average spins ranging from $34 \hbar$ to $55 \hbar$.

The comparison between the $a_{2}$ coefficient of panels (a) and (b) of Fig. 9 shows a larger $a_{2}$ absolute value for the charge-asymmetric reaction in the DD energy region, indicating a more anisotropic $\gamma$-ray angular distribution. In panel (c) we show the $\gamma$-ray angular distribution of the two reactions integrated over energy from 9 to $15 \mathrm{MeV}$ and corrected for the detection efficiency (symbols), where the DD obtains its maximum value [see the percent difference between the $\gamma$ spectra of the two reactions in panel (c) of Fig. 8]. From a best fit to the data, shown with a solid (dashed) line for the ${ }^{40} \mathrm{Ca}+{ }^{152} \mathrm{Sm}\left({ }^{48} \mathrm{Ca}+{ }^{144} \mathrm{Sm}\right)$ reaction, we obtain $a_{2}=-0.13 \pm 0.03\left(a_{2}=-0.06 \pm 0.02\right)$ for the ${ }^{40} \mathrm{Ca}+{ }^{152} \mathrm{Sm}\left({ }^{48} \mathrm{Ca}+{ }^{144} \mathrm{Sm}\right)$ reaction. By using the same argument as previously for the spectra, the observed difference in the $\gamma$-ray angular distribution of the two systems can only be ascribed to entrance channel effects, namely the DD excitation.

In panel (d) of Fig. 9 we display the angular distribution of the difference between the data of panel (c). This is the DD $\gamma$-ray angular distribution, energy integrated from 9 to $15 \mathrm{MeV}$. The DD $\gamma$-ray angular distribution provides important information, because it is a sensitive probe of the fusion dynamics and of the DD lifetime. Indeed, the amount of anisotropy, if present, is related to the interplay of the rotation angular velocity of the dinuclear system during the prompt DD emission and the instant at which this emission occurs. For a more extended discussion, see Refs. [17,18,21]. From the figure we note that the DD $\gamma$-ray angular distribution is very anisotropic around $90^{\circ}$ and it can be reproduced well by using an anisotropy coefficient $a_{2}=-1$ (solid line) that results in an angular distribution of the $\sin ^{2}\left(\theta_{\gamma}\right)$ form of emission from a dipole oscillation along the beam axis (solid line).

We recall here that the DD oscillation is expected to occur along the symmetry axis of the dinuclear system which, for near-central collisions, forms a relatively small angle with the beam axis at the very early moments of its formation. In the present experiment where evaporation residues are considered, the maximum angle between the DD oscillation axis and that of the beam at the early moments of the dinuclear system formation is $\sim 10^{\circ}$ for an interaction radius of $R=10.6 \mathrm{fm}\left(r_{0}=1.2 \mathrm{fm}\right.$ was considered) and a maximum impact parameter for evaporation $b_{\max } \sim 2 \mathrm{fm}$. In the case of a larger mean inclination of the axis of the DD oscillation, because rotation has taken place meanwhile, we would expect a widening of the angular distribution with respect to $90^{\circ}$. This means a more isotropic angular distribution like that shown with the dotted line in Fig. 9(d), obtained by using an $a_{2}=-0.25$. According to the previous considerations, the observed large anisotropy proves the preequilibrium nature of the DD $\gamma$ emission, in agreement with our previous result for a composite system in the ${ }^{132} \mathrm{Ce}$ mass region $[17,18]$.

The DD $\gamma$ yield integrated over angle and over energy from 9 to $15 \mathrm{MeV}$ was obtained from the best fit to the data, shown in panel (d) of Fig. 9, by using $a_{2}=-1$ (solid line in the figure). To have the yield, $M_{\gamma, \mathrm{DD} \text {,exp }}$, integrated from 8 to $21 \mathrm{MeV}$ and to compare it with existing results integrated in the same energy range, we made use of the Lorentzian curve that reproduces the experimental DD $\gamma$-ray spectrum [shown in panel (b) of Fig. 8] to recover the factor between the two integrals: from 9-15 MeV to $8-21 \mathrm{MeV} . M_{\gamma, \mathrm{DD} \text {,exp }}$ and the associated error are shown in Table VII.

We can compare the present result with those obtained in our earlier works, where colliding systems leading to a ${ }^{132} \mathrm{Ce}$ 

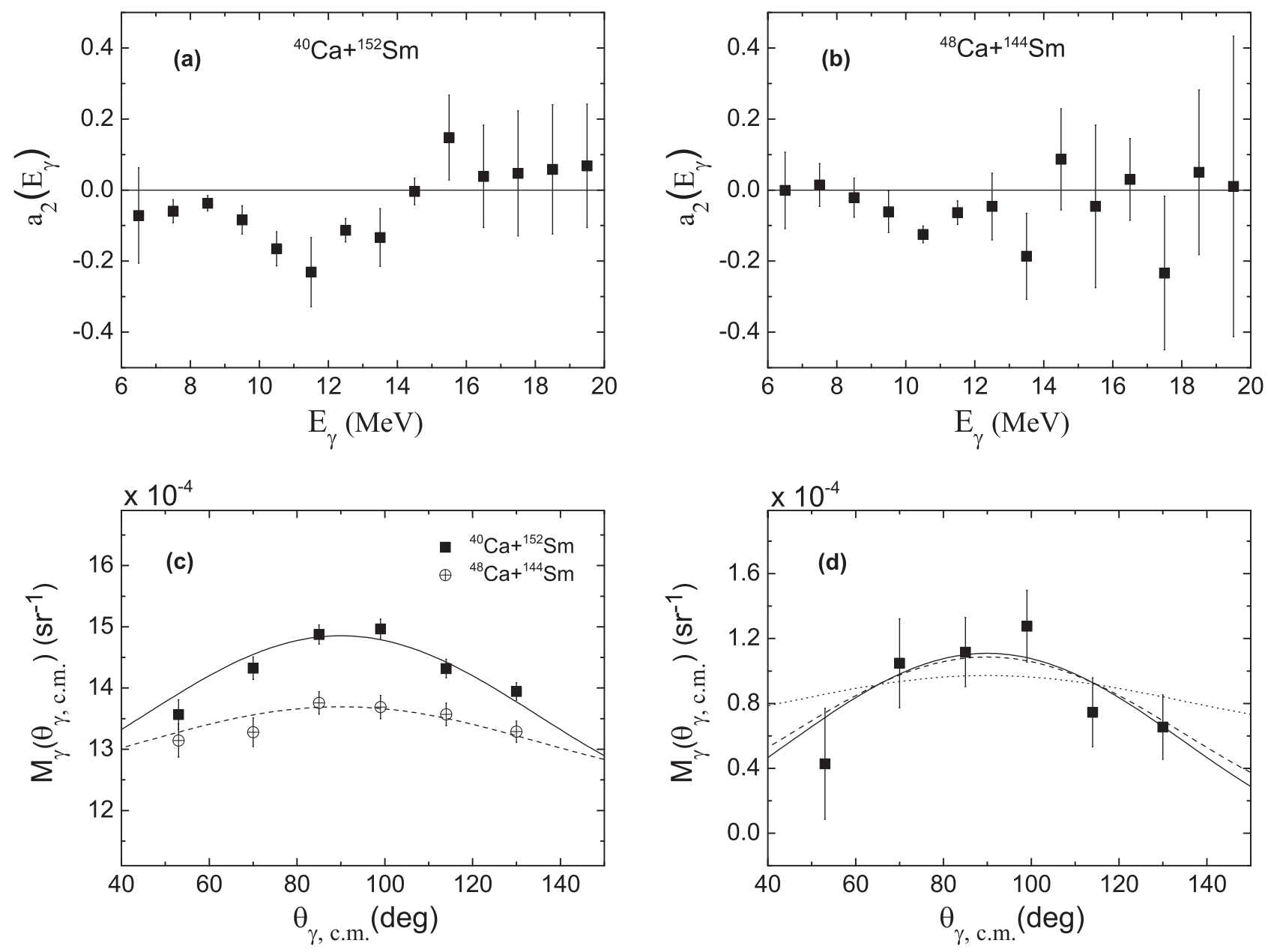

FIG. 9. Energy dependence of the $a_{2}$ coefficient for (a) the ${ }^{40} \mathrm{Ca}+{ }^{152} \mathrm{Sm}$ and (b) the ${ }^{48} \mathrm{Ca}+{ }^{144} \mathrm{Sm}$ system. (c) $9 \mathrm{MeV} \leqslant E_{\gamma} \leqslant 15 \mathrm{MeV}$ $\gamma$-ray angular distribution of the ${ }^{40} \mathrm{Ca}+{ }^{152} \mathrm{Sm}$ (solid symbols) and ${ }^{48} \mathrm{Ca}+{ }^{144} \mathrm{Sm}$ (open symbols) reactions, corrected for the detection efficiency. The solid and dashed lines represent a fit of the data with a Legendre polynomial expansion $\left(a_{2}\right.$ coefficients given in the text). (d) Angular distribution of the difference between the data of the two reactions appearing in panel (c), i.e., DD $\gamma$-ray angular distribution energy-integrated in the interval $9 \mathrm{MeV} \leqslant E_{\gamma} \leqslant 15 \mathrm{MeV}$ (symbols). The solid line is the result of a fit of the DD angular distribution with a Legendre polynomial expansion by using $a_{2}=-1$, the dashed line is a fit with $a_{2}=-0.84$ obtained within the BNV framework with the asystiff choice, and the dotted line shows the fit with an $a_{2}=-0.25$. All the quantities are in the center-of-mass reference frame.

compound nucleus were studied at various $E_{\text {lab }}$ ranging from 6 to $16 \mathrm{MeV} /$ nucleon by employing the difference method technique (see, for instance, Fig. 15 of Ref. [18]). Those colliding systems correspond to a similar mass asymmetry but to a lower compound nucleus mass and to a lower initial dipole moment than that of the ${ }^{40} \mathrm{Ca}+{ }^{152} \mathrm{Sm}$ reaction studied here. We notice that the DD yield extracted in the ${ }^{40} \mathrm{Ca}+{ }^{152} \mathrm{Sm}$ reaction at $E_{\text {lab }}=11 \mathrm{MeV} /$ nucleon, does not increase as expected with the initial dipole moment, because it is similar to the yield obtained in the ${ }^{32} \mathrm{~S}+{ }^{100}$ Mo reaction at $E_{\mathrm{lab}}=9 \mathrm{MeV} /$ nucleon. However, as already claimed in the Introduction, the DD yield is influenced by the interplay of several factors including beam energy, impact parameter, mass asymmetry, mass of the colliding ions or competition with other reaction mechanisms. It seems therefore, that some other reaction parameter counteracts the expected DD yield increase with the initial dipole moment. This point is further discussed in the next section, where possible explanations and some suggestions are presented.

\section{BNV CALCULATIONS AND DISCUSSION}

Several theoretical models were used to investigate the properties of the DD excitation [3-9,21]. In particular, calculations within a BNV transport model, based on a collective bremsstrahlung analysis of the entrance channel reaction dynamics [8,21], were widely adopted to describe the DD $\gamma$ decay. This last one is the approach we have chosen to use here to describe how the DD develops in the charge-asymmetric channel ${ }^{40} \mathrm{Ca}+{ }^{152} \mathrm{Sm}$ at $11 \mathrm{MeV} /$ nucleon. The evolution of the dinuclear system along the fusion path, including the isovector degrees of freedom, is described self-consistently by the behavior of the one-body distribution function for protons and neutrons, $f_{p}(\mathbf{r}, \mathbf{p}, t), f_{n}(\mathbf{r}, \mathbf{p}, t)$, as ruled by the 
density-dependent mean field. In the transport simulations no free parameters were used and for the potential part of the symmetry energy two different density parametrizations were tested: the asysoft and the asystiff EOS. For the asysoft EOS, the symmetry energy has a weak density dependence close to the saturation, with an almost flat behavior at subsaturation densities. For the asystiff EOS choice, the interaction part of the symmetry term displays a linear density dependence. For details in the calculations, see, for instance, [21] and references therein. To reduce the numerical noise, we run ten events for each set of macroscopic initial conditions and the displayed quantities are the averages over this ensemble.

We summarize in Table VII the DD parameters extracted with the asystiff EOS choice (in parentheses those obtained with the asysoft one are given): centroid energy $E_{\mathrm{DD} \text {,th }}$, width $\Gamma_{\mathrm{DD}, \mathrm{th}}$, and yield $M_{\gamma, \mathrm{DD}, \mathrm{th}}$. The yield was obtained by integrating over angle and over energy in the resonance region for each impact parameter and by summing over the impact parameters with the corresponding geometrical weights. The impact parameter window considered for the calculation of $E_{\mathrm{DD}, \mathrm{th}}, \Gamma_{\mathrm{DD} \text {,th }}$, and $M_{\gamma, \mathrm{DD} \text {,th }}$ is taken from 0 up to the $b_{\max }$ value indicated in the last column of Table VII. Values for $b_{\max }=2 \mathrm{fm}$, shown in the first row, are associated with the most central collisions and, thus, with evaporation events that are of interest in the present work. For comparison purposes, the yield was calculated also for higher impact parameters: up to $3 \mathrm{fm}$ that corresponds approximately to $J_{B_{f}=0}$, the maximum spin for compound nucleus formation (second row), and up to 4 and $6 \mathrm{fm}$ in the third and fourth row, respectively.

The centroid energy $E_{\mathrm{DD} \text {,th }}$ was deduced from the power spectrum of the dipole moment for evaporation events. We notice here that the centroid energy obtained within an identical theoretical framework, but exciting the GDR mode from the ground state of ${ }^{192} \mathrm{~Pb}$ as described in [70], was located at $E_{\mathrm{GDR}, \mathrm{th}}=10.9$ (12.4) $\mathrm{MeV}$ for the asystiff (asysoft) choice. A shift of the DD centroid energy (see Table VII) of around 2.6 (2.6) $\mathrm{MeV}$ to lower values with respect to that of the GDR is a clear indication that the DD $\gamma$ emission took place when the system was still elongated along an axial-symmetry axis, i.e., before shape equilibration [71]. This result is in good agreement with our experimental finding. The width $\Gamma_{\mathrm{DD}, \mathrm{th}}$, was derived from a fit of the dipole moment obtained from the numerical calculations with an oscillating damped function and agrees also with the experimental one.

By averaging over impact parameters up to $b_{\max }=2 \mathrm{fm}$, the anisotropy coefficient of the DD $\gamma$ rays was found to be equal to $a_{2}=-0.84(-0.82)$ with the asystiff (asysoft) choice. This value results in a very anisotropic angular distribution that is compatible (within errors) with the experimental one, as can be seen by the dashed line in panel (d) of Fig. 9. As mentioned previously, such an $a_{2}$ value indicates a preferential oscillation axis of the DD, triggered at the early stage of the fusion path, along an axis that has not rotated much on the reaction plane during the DD lifetime.

By looking at the theoretical DD yield obtained with both EOS choices, we notice that it overpredicts significantly the data, well outside the experimental uncertainties. The theoretical yield is still larger than the experimental one even if we consider integration up to $6 \mathrm{fm}$ that is a toohigh impact parameter for fusion evaporation to occur. For such high-impact parameters, moreover, the theoretical DD angular distribution becomes much more isotropic, no longer compatible with our results.

BNV calculations reproduced better the DD yield for colliding systems leading to the ${ }^{132} \mathrm{Ce}$ composite system and having lower initial dipole moment than that of the ${ }^{40} \mathrm{Ca}+{ }^{152} \mathrm{Sm}$ reaction [18]. In that case, the data were found to be in good agreement with calculations at incident energies of 6 and $16 \mathrm{MeV} /$ nucleon, while the experimental yield at $9 \mathrm{MeV} /$ nucleon differed by at most $40 \%$ from the calculated one. In the case of the ${ }^{40} \mathrm{Ca}+{ }^{152} \mathrm{Sm}$ reaction, the disagreement is not understood in the framework of the BNV model. The results of the present work suggest that BNV calculations do not take into account some aspects of the reaction dynamics which could inhibit the preequilibrium $\gamma$-ray emission. An ingredient neglected in the present calculations is the deformation of the ${ }^{152} \mathrm{Sm}$ target ground state that could influence the DD excitation mechanism.

By examining other theoretical models in the literature, it is worth noticing that time-dependent Hartree-Fock calculations coupled to the phonon model [4] foresee a lower DD $\gamma$ yield with increasing the mass of the colliding ions, attributed to the fact that reactions with light nuclei are less damped than those involving more nucleons. However, some comments about the phonon model $[1,2]$ results are in order. The DD $\gamma$ yield obtained within this model is systematically below the bremsstrahlung evaluation, as has been shown in Ref. [8]. This is related to the uncertainties in the estimation of the number of preequilibrium GDR phonons present when the compound nucleus is formed, overcome by the bremsstrahlung method [8] that we used for comparison in the paper. Thus, while the phonon model results could suggest a better agreement with our data, the above-mentioned uncertainties make this theoretical prediction less accurate than that obtained with the bremsstrahlung approach. It is clear, therefore, that further investigation is needed from both the theoretical and the experimental sides to shed light on the origin of the observed discrepancy between data and BNV calculations.

The observation of the DD preequilibrium $\gamma$ radiation for the first time in the fusion-evaporation decay channel of a heavy composite system encourages in exploring the possibility to use it as a fast cooling mechanism in fusion reactions. At low excitation energy, relevant for the formation of superheavy elements, this could increase significantly the compound nucleus survival probability against fission, favoring the formation of evaporation residues that can be studied via their subsequent $\alpha$ decay and spontaneous fission. In hot fusion reactions [72], which is one of the two employed experimental approaches to synthesize superheavy elements, the compound nucleus is formed at excitation energy of 30-60 MeV, where statistical fission largely dominates over neutron evaporation. Nonetheless dissipative effects may retard fission [73], assisting cooling of the nucleus. Such dissipative effects were observed in studies of pre-scission neutrons [74] and pre-scission GDR $\gamma$ rays [75] from hot heavy systems. In this context, cooling through emission of preequilibrium DD $\gamma$ radiation in charge-asymmetric hot 
fusion reactions could allow the composite system to reach the statistical phase with a lower excitation energy, resulting in a larger superheavy-element formation probability.

The scenario outlined above was investigated theoretically in Ref. [10] for the ${ }^{265} \mathrm{Hs}(Z=108)$ nucleus formed in the ${ }^{124} \mathrm{Xe}+{ }^{141} \mathrm{Xe}$ charge-asymmetric reaction at $E^{*}=54 \mathrm{MeV}$. An enhancement of the total survival probability of the compound nucleus against fission owing solely to the charge asymmetry in the entrance channel of a factor six was obtained. This estimation was done in the framework of a "hybrid" statistical model of the compound nucleus decay. The preequilibrium $\gamma$-ray emission was externally introduced while the fission width evolution was calculated with the diffusion model for fusion-fission dynamics described in Ref. [76]. From an experimental point of view, to verify a potential usefulness of the DD $\gamma$ radiation in the superheavy-element formation, we considered it mandatory, first of all, to investigate its existence in fusion-evaporation events of systems heavier than those studied previously. Our data prove that the DD survives in such a heavy system and decays emitting preequilibrium $\gamma$-rays, though with a lower multiplicity than predicted in the framework of BNV calculations. A lower multiplicity implies a lower enhancement of the compound nucleus survival probability owing to the entrance-channel charge asymmetry (see Eq. (24) of Ref. [10]). Nevertheless, the enhancement still exists, allowing us to consider that the experimental findings of this paper provide, even if higher incident energies and still-too-low masses were investigated, the first experimental data useful to this aim.

\section{CONCLUSIONS}

In conclusion, we searched for the DD $\gamma$ radiation in fusionevaporation events for the first time in a composite system heavier than those studied so far, in the $A \sim 190$ mass region. Two reactions, differing in the charge asymmetry between the colliding ions, were employed to form the same compound nucleus at identical initial conditions: the charge-asymmetric ${ }^{40} \mathrm{Ca}+{ }^{152} \mathrm{Sm}$ and the nearly charge-symmetric ${ }^{48} \mathrm{Ca}+{ }^{144} \mathrm{Sm}$. The multiplicity spectra and the angular distribution of the light charged particles emitted in these reactions were used to determine the average mass, average charge, and average excitation energy of the compound nucleus.

First, we presented new results on the $\gamma$ decay of the GDR thermally excited in the ${ }^{189} \mathrm{Hg}$ compound nucleus formed at $E^{*}=220.5 \mathrm{MeV}$ and in its evaporation daughters by studying the $\gamma$-ray multiplicity spectrum and the $\gamma$-ray angular distribution relative to the nearly charge-symmetric reaction. The $\gamma$ spectrum was analyzed in the framework of the statistical model, implementing various prescriptions of the level density parameter in the CASCADE code. We extracted a GDR centroid energy that is in agreement with the ground-state value and a width that increases with the temperature of the nuclear states on which the GDR is built. Moreover, no cutoff in the GDR $\gamma$-ray emission was needed to reproduce our data, in agreement with expectations for this mass region at excitation energy $E^{*}=1.2 \mathrm{MeV} /$ nucleon. The angular distribution of the statistical GDR $\gamma$ rays showed a small anisotropy around $90^{\circ}$ with respect to that of a stretched dipole transition, denoting a rather large attenuation of the anisotropy coefficient. This was attributed to orientation fluctuations of the nuclear spin vector that become important at low rotational frequencies, as in the present case.

By comparing the $\gamma$-ray multiplicity spectra and $\gamma$-ray angular distributions of the two studied reactions with each other, we evidenced the DD excitation in the composite system of the more charge-asymmetric channel in a model-independent way. We extracted the spectrum and angular distribution of the DD $\gamma$ decay by subtracting the data of the charge-symmetric channel from those of the charge-asymmetric one. The centroid energy of the DD $\gamma$ spectrum was found to be $3 \mathrm{MeV}$ lower than that of the ground-state GDR, while the DD $\gamma$-ray angular distribution was much more anisotropic than that of the GDR in the hot compound nucleus. These results indicate that (i) the DD $\gamma$ emission took place during the early moments of the reaction when the system was still elongated along the symmetry axis, i.e., before shape equilibration; (ii) the DD oscillation axis had not rotated much with respect to the beam direction in the reaction plane, resulting in a very anisotropic angular distribution. Both elements indicate that such a radiation occurs in a preequilibrium phase of the composite system lifetime. The lowering of the experimental DD centroid energy with respect to that of the ground-state GDR and the experimental DD width and angular distribution were found to be in good agreement with the theoretical ones obtained by employing a BNV transport model, based on a collective bremsstrahlung analysis of the reaction dynamics. However, the theoretical DD yield overpredicted the data, suggesting that BNV calculations do not take into account some aspects of the reaction dynamics and calling for further investigation to clarify this aspect.

Finally, we discussed the possibility to use the DD preequilibrium $\gamma$ emission as a cooling mechanism of the composite system along the fusion path, before reaching the equilibrium phase. This could increase the formation probability of the compound nucleus when very heavy colliding ions are involved. The present work makes it possible to take a step forward in the study of superheavy-element formation, demonstrating that the DD $\gamma$ radiation survives in heavy composite systems, though with a lower multiplicity than predicted with BNV calculations. To predict evaporation residue cross sections by taking into account the isospin degree of freedom, an appropriate theoretical model is needed together with data on the DD energy- and angle-integrated multiplicity in heavy composite systems formed under various reaction conditions. Besides the superheavy-element question, the DD prompt radiation emitted in radioactive-ion-beam-induced reactions, along with well-tested theoretical models, will allow us to probe the density dependence of the symmetry energy in the equation of state at nuclear densities lower than the saturation one, where the dynamical dipole mode is active [21].

\section{ACKNOWLEDGMENT}

V.B. was supported by a grant from the Romanian National Authority for Scientific Research, CNCSUEFISCDI, Project No. PN-II-ID-PCE-2011-3-0972. 
[1] D. Brink, Nucl. Phys. A 519, 3 (1990).

[2] Ph. Chomaz, M. Di Toro, and A. Smerzi, Nucl. Phys. A 563, 509 (1993).

[3] V. Baran, M. Cabibbo, M. Colonna, M. Di Toro, and N. Tsoneva, Nucl. Phys. A 679, 373 (2001).

[4] C. Simenel, Ph. Chomaz, and G. de France, Phys. Rev. Lett. 86, 2971 (2001).

[5] A. S. Umar and V. E. Oberacker, Phys. Rev. C 76, 014614 (2007).

[6] M. Papa, W. Tian, G. Giuliani, F. Amorini, G. Cardella, A. Di Pietro, P. P. Figuera, G. Lanzalone, S. Pirrone, F. Rizzo, and D. Santonocito, Phys. Rev. C 72, 064608 (2005).

[7] H. L. Wu, W. D. Tian, Y. G. Ma, X. Z. Cai, J. G. Chen, D. Q. Fang, W. Guo, and H. W. Wang, Phys. Rev. C 81, 047602 (2010); Y. G. Ma, G. H. Liu, X. Z. Cai, D. Q. Fang, W. Guo, W. Q. Shen, W. D. Tian, and H. W. Wang, ibid. 85, 024618 (2012); S. Q. Ye, X. Z. Cai, Y. G. Ma, and W. Q. Shen, ibid. 88, 047602 (2013).

[8] V. Baran, D. M. Brink, M. Colonna, and M. Di Toro, Phys. Rev. Lett. 87, 182501 (2001).

[9] M. Cabibbo, Phys. Rev. C 77, 014608 (2008).

[10] C. Simenel, Ph. Chomaz, and G. de France, Phys. Rev. C 76, 024609 (2007).

[11] D. Pierroutsakou, M. Di Toro, F. Amorini, V. Baran, A. Boiano, A. De Rosa, A. D’Onofrio, G. Inglima, M. La Commara, A. Ordine, N. Pellegriti, F. Rizzo, V. Roca, M. Romoli, M. Sandoli, M. Trotta, and S. Tudisco, Eur. Phys. J. A 16, 423 (2003); Nucl. Phys. A 687, 245 (2001).

[12] F. Amorini, G. Cardella, A. Di Pietro, P. Figuera, G. Lanzalone, Lu Jun, A. Musumarra, M. Papa, S. Pirrone, F. Rizzo, W. Tian, and S. Tudisco, Phys. Rev. C 69, 014608 (2004).

[13] S. Flibotte, Ph. Chomaz, M. Colonna, M. Cromaz, J. DeGraaf, T. E. Drake, A. Galindo-Uribarri, V. P. Janzen, J. Jonkman, S. W. Marshall, S. M. Mullins, J. M. Nieminen, D. C. Radford, J. L. Rodriguez, J. C. Waddington, D. Ward, and J. N. Wilson, Phys. Rev. Lett. 77, 1448 (1996).

[14] M. Cinausero et al., Il Nuovo Cimento A 111A, 613 (1998).

[15] D. Pierroutsakou, A. Boiano, A. De Rosa, M. Di Pietro, G. Inglima, M. La Commara, Ruhan Ming, B. Martin, R. Mordente, A. Ordine, F. Rizzo, V. Roca, M. Romoli, M. Sandoli, F. Soramel, L. Stroe, M. Trotta, and E. Vardaci, Eur. Phys. J. A 17, 71 (2003).

[16] D. Pierroutsakou, B. Martin, G. Inglima, A. Boiano, A. De Rosa, M. Di Pietro, M. La Commara, R. Mordente, M. Romoli, M. Sandoli, M. Trotta, E. Vardaci, T. Glodariu, M. Mazzocco, C. Signorini, L. Stroe, V. Baran, M. Colonna, M. Di Toro, and N. Pellegriti, Phys. Rev. C 71, 054605 (2005).

[17] B. Martin et al., Phys. Lett. B 664, 47 (2008).

[18] D. Pierroutsakou et al., Phys. Rev. C 80, 024612 (2009).

[19] A. Corsi et al., Phys. Lett. B 679, 197 (2009).

[20] A. Giaz et al., Phys. Rev. C 90, 014609 (2014).

[21] V. Baran, C. Rizzo, M. Colonna, M. Di Toro, and D. Pierroutsakou, Phys. Rev. C 79, 021603 (2009).

[22] F. Puhlhofer, Nucl. Phys. A 280, 267 (1977); M. N. Harakeh (private communication, extended version).

[23] A. Gavron, Phys. Rev. C 21, 230 (1980).

[24] A. J. Sierk, Phys. Rev. C 33, 2039 (1986).

[25] E. Migneco, C. Agodi, R. Alba, G. Bellia, R. Coniglione, A. Del Zoppo, P. Finocchiaro, C. Maiolino, P. Piattelli, G. Raia, and P. Sapienza, Nucl. Instrum. Methods Phys. Res., Sect. A 314, 31 (1992).
[26] T. Matulewicz, E. Grosse, H. Emling, H. Grein, and R. Kulessa, Nucl. Instrum. Methods Phys. Res., Sect. A 274, 501 (1989).

[27] S. Kubota, T. Motobayashi, M. Ogiwara, H. Murakami, Y. Ando, J. Ruan, and S. Shirato, Nucl. Instrum. Methods Phys. Res., Sect. A 285, 436 (1989).

[28] A. Del Zoppo, C. Agodi, R. Alba, G. Bellia, R. Coniglione, P. Finocchiaro, C. Maiolino, E. Migneco, A. Peghaire, P. Piattelli, and P. Sapienza, Nucl. Instrum. Methods Phys. Res., Sect. A 327, 363 (1993).

[29] H. Morgenstern, W. Bohne, W. Galster, K. Grabisch, and A. Kyanowski, Phys. Rev. Lett. 52, 1104 (1984).

[30] M. P. Kelly, J. F. Liang, A. A. Sonzogni, K. A. Snover, J. P. S. van Schagen, and J. P. Lestone, Phys. Rev. C 56, 3201 (1997).

[31] E. Holub, D. Hilscher, G. Ingold, U. Jahnke, H. Orf, and H. Rossner, Phys. Rev. C 28, 252 (1983).

[32] E. Migneco, C. Agodi, R. Alba, G. Bellia, R. Coniglione, A. Del Zoppo, P. Finocchiaro, C. Maiolino, P. Piattelli, G. Russo, P. Sapienza, A. Badalà, R. Barbera, A. Palmeri, G. S. Pappalardo, F. Riggi, A. C. Russo, A. Perghaire, and A. Bonasera, Phys. Lett. B 298, 46 (1993).

[33] D. G. d'Enterria, G. Martínez, L. Aphecetche, H. Delagrange, F. Fernández, H. Löhner, R. Ortega, R. Ostendorf, Y. Schutz, and H. W. Wilschut, Phys. Lett. B 538, 27 (2002).

[34] H. Nifenecker and J. A. Pinston, Annu. Rev. Nucl. Part. Sci. 40, 113 (1990).

[35] B. L. Berman and S. C. Fultz, Rev. Mod. Phys. 47, 713 (1975).

[36] S. S. Dietrich and B. L. Berman, At. Data Nucl. Data Tables 38, 199 (1988).

[37] M. Gallardo, M. Diebel, T. Døssing, and R. A. Broglia, Nucl. Phys. A 443, 415 (1985).

[38] R. Butsch, M. Thoennessen, D. R. Chakrabarty, M. G. Herman, and P. Paul, Phys. Rev. C 41, 1530 (1990).

[39] D. R. Chakrabarty, M. Thoennessen, N. Alamanos, P. Paul, and S. Sen, Phys. Rev. Lett. 58, 1092 (1987).

[40] M. Thoennessen, D. R. Chakrabarty, R. Butsch, M. G. Herman, P. Paul, and S. Sen, Phys. Rev. C 37, 1762 (1988).

[41] D. R. Chakrabarty, V. M. Datar, R. K. Choudhury, B. K. Nayak, Y. K. Agarwal, C. V. K. Baba, and M. K. Sharan, Phys. Rev. C 53, 2739 (1996).

[42] E. Ramakrishnan, A. Azhari, J. R. Beene, R. J. Charity, M. L. Halbert, P. F. Hua, R. A. Kryger, P. E. Mueller, R. Pfaff, D. G. Sarantites, L. G. Sobotka, M. Thoennessen, G. Van Buren, R. L. Varner, and S. Yokoyama, Phys. Lett. B 383, 252 (1996).

[43] D. Kusnezov, Y. Alhassid, and K. A. Snover, Phys. Rev. Lett. 81, 542 (1998).

[44] F. Plasil and M. Blann, Phys. Rev. C 11, 508 (1975).

[45] A. Stefanini et al., Eur. Phys. J. A 23, 473 (2005); M. Trotta et al., ibid. 25, 615 (2005).

[46] D. J. Hinde, J. R. Leigh, J. O. Newton, W. Galster, and S. Sie, Nucl. Phys. A 385, 109 (1982).

[47] J. B. Natowitz, R. Wada, K. Hagel, T. Keutgen, M. Murray, A. Makeev, L. Qin, P. Smith, and C. Hamilton, Phys. Rev. C 65, 034618 (2002).

[48] B. J. Fineman, K.-T. Brinkmann, A. L. Caraley, N. Gan, R. L. McGrath, and J. Velkovska, Phys. Rev. C 50, 1991 (1994).

[49] A. L. Caraley, B. P. Henry, J. P. Lestone, and R. Vandenbosch, Phys. Rev. C 62, 054612 (2000).

[50] R. J. Charity, Phys. Rev. C 82, 014610 (2010).

[51] W. Reisdorf, Z. Phys. A: At. Nucl. 300, 227 (1981).

[52] A. V. Ignatyuk, G. N. Smirenkin, and A. S. Tishin, Sov. J. Nucl. Phys. 21, 255 (1975). 
[53] M. Ciemala et al., Phys. Rev. C 91, 054313 (2015).

[54] W. E. Ormand, P. F. Bortignon, A. Bracco, and R. A. Broglia, Phys. Rev. C 40, 1510 (1989).

[55] M. Kicinska-Habior, K. A. Snover, J. A. Behr, G. Feldman, C. A. Gossett, and J. H. Gundlach, Phys. Rev. C 41, 2075 (1990).

[56] W. D. Myers, Droplet Model of Atomic Nuclei (IFI/Plenum, New York, 1977).

[57] G. Bellia, R. Alba, R. Coniglione, A. Del Zoppo, P. Finocchiaro, C. Maiolino, E. Migneco, P. Piattelli, P. Sapienza, N. Frascaria, I. Lhenry, J. C. Roynette, T. Suomijärvi, N. Alamanos, F. Auger, A. Gillibert, D. Pierroutsakou, J. L. Sida, and P. R. Silveira Gomes, Nucl. Instrum. Methods Phys. Res., Sect. A 329, 173 (1993).

[58] D. Santonocito, Y. Blumenfeld, C. Agodi, R. Alba, G. Bellia, R. Coniglione, F. Delaunay, A. Del Zoppo, P. Finocchiaro, F. Hongmei, V. Lima, C. Maiolino, E. Migneco, P. Piattelli, P. Sapienza, J. A. Scarpaci, and O. Wieland, Phys. Rev. C 90, 054603 (2014).

[59] H. Sagawa and G. F. Bertsch, Phys. Lett. B 146, 138 (1984).

[60] M. Barranco, A. Polls, S. Marcos, J. Navarro, and J. Treiner, Phys. Lett. B 154, 96 (1985).

[61] E. Lipparini and S. Stringari, Nucl. Phys. A 482, 205 (1988).

[62] F. Garcias, M. Barranco, J. Navarro, and E. Suraud, Z. Phys. A 337, 261 (1990).

[63] D. Kusnezov and W. E. Ormand, Phys. Rev. Lett. 90, 042501 (2003).
[64] J. J. Gaardhøje, A. M. Bruce, J. D. Garrett, B. Herskind, D. Barnéoud, M. Maurel, H. Nifenecker, J. A. Pinston, P. Perrin, C. Ristori, F. Schussler, A. Bracco, and M. Pignanelli, Phys. Rev. Lett. 59, 1409 (1987).

[65] T. Suomijärvi et al., Phys. Rev. C 53, 2258 (1996).

[66] M. E. Rose, Phys. Rev. 91, 610 (1953).

[67] K. A. Snover, Annu. Rev. Nucl. Part. Sci. 36, 545 (1986).

[68] Y. Alhassid and B. Bush, Phys. Rev. Lett. 65, 2527 (1990).

[69] M. Mattiuzzi, A. Bracco, F. Camera, B. Million, M. Pignanelli, J. J. Gaardhøje, A. Maj, T. Ramsøy, T. Tveter, and Z. Źelazny, Phys. Lett. B 364, 13 (1995).

[70] V. Baran, M. Colonna, M. Di Toro, A. Croitoru, and D. Dumitru, Phys. Rev. C 88, 044610 (2013).

[71] V. Baran, M. Colonna, M. Di Toro, A. Guarnera, and A. Smerzi, Nucl. Phys. A 600, 111 (1996).

[72] Y. T. Oganessian et al., Phys. Rev. C 70, 064609 (2004).

[73] H. A. Kramers, Physica 7, 284 (1940).

[74] R. Yanez, W. Loveland, L. Yao, J. S. Barrett, S. Zhu, B. B. Back, T. L. Khoo, M. Alcorta, and M. Albers, Phys. Rev. Lett. 112, 152702 (2014).

[75] T. S. Tveter, J. J. Gaardhøje, A. Maj, T. Ramsøy, A. Ataç, J. Bacelar, A. Bracco, A. Buda, F. Camera, B. Herskind, W. Korten, W. Królas, A. Menthe, B. Million, H. Nifenecker, M. Pignanelli, J. A. Pinston, H. v. d. Ploeg, F. Schussler, and G. Sletten, Phys. Rev. Lett. 76, 1035 (1996).

[76] Y. Aritomo, T. Wada, M. Ohta, and Y. Abe, Phys. Rev. C 59, 796 (1999). 